\title{
Strict Local Martingales and Optimal Investment in a Black-Scholes Model with a Bubble*
}

\author{
Martin Herdegen ${ }^{\dagger} \quad$ Sebastian Herrmann ${ }^{\ddagger}$
}

November 8, 2017

\begin{abstract}
There are two major streams of literature on the modeling of financial bubbles: the strict local martingale framework and the Johansen-Ledoit-Sornette (JLS) financial bubble model. Based on a class of models that embeds the JLS model and can exhibit strict local martingale behavior, we clarify the connection between these previously disconnected approaches. While the original JLS model is never a strict local martingale, there are relaxations which can be strict local martingales and which preserve the key assumption of a log-periodic power law for the hazard rate of the time of the crash. We then study the optimal investment problem for an investor with constant relative risk aversion in this model. We show that for positive instantaneous expected returns, investors with relative risk aversion above one always ride the bubble.
\end{abstract}

Keywords Bubbles; Strict local martingales; JLS model; Optimal investment; Utility maximization; Power utility.

AMS MSC 2010 Primary, 91G10; Secondary, 49N15, 49J15.

JEL Classification G11, C61.

\section{Introduction}

Financial bubbles are often associated with a disparity between the price of an asset and its "fundamental value" (see Kaizoji \& Sornette, 2010; Protter, 2013; Scherbina \& Schlusche, 2014). It has been argued in the mathematical finance literature that this form of mispricing can be captured very generally by modeling asset prices as processes that are strict local martingales (i.e., local martingales that are not martingales) under some equivalent local martingale measure (ELMM); see Loewenstein \& Willard (2000), Cox \& Hobson (2005), Heston et al. (2007), Jarrow et al. (2007, 2010), Protter (2013), and the references therein. Another strand of the literature on financial bubbles originated from the idea of fitting asset prices to a so-called log-periodic power law in order to detect and predict the end of possible bubbles (Bouchaud et al. , 1996; Feigenbaum $\&$ Freund, 1996). This led to the development of the Johansen-Ledoit-Sornette (JLS) financial bubble model (Johansen \& Sornette, 1999a; Johansen et al. , 2000). However, the JLS model is a martingale by definition and does not mention strict local martingales at all.

This article has two objectives: (1) to clarify the connection between these previously disconnected modeling approaches and (2) to analyze how a rational investor would act in the presence of an asset price bubble of a generalized JLS type.

\footnotetext{
* The authors thank Jérôme Detemple, David Hobson, Johannes Muhle-Karbe, Rémy Praz, and, in particular, Martin Schweizer for stimulating discussions and comments. We would also like to thank an associate editor and the referees for helpful comments. Moreover, we gratefully acknowledge financial support by the Swiss Finance Institute and by the National Centre of Competence in Research "Financial Valuation and Risk Management" (NCCR FINRISK), Project D1 (Mathematical Methods in Financial Risk Management). The NCCR FINRISK is a research instrument of the Swiss National Science Foundation.

${ }^{\dagger}$ Department of Statistics, University of Warwick, Coventry, CV4 7AL, UK, email M.Herdegen@warwick.ac.uk.

${ }_{\ddagger}^{\ddagger}$ Department of Mathematics, University of Michigan, 530 Church Street, Ann Arbor, MI 48109, USA, email sherrma@umich.edu.
}

This is the author manuscript accepted for publication and has undergone full peer review but has not been through the copyediting, typesetting, pagination and proofreading process, which may lead to differences between this version and the Version of Record. Please cite this article as doi: $10.1111 /$ mafi.12175

This article is protected by copyright. All rights reserved. 
The Johansen-Ledoit-Sornette model. The JLS model proposes ${ }^{1}$ that the price process of a financial asset can be modeled as the sum of its "fundamental value" (which is not further specified) and a bubble component $S=\left(S_{t}\right)_{t \in[0, T]}$ which has the dynamics

$$
\frac{\mathrm{d} S_{t}}{S_{t-}}=\phi^{\prime}(t) \mathrm{d} t+\sigma \mathrm{d} W_{t}-\delta \mathrm{d} J_{t}
$$

where $\phi^{\prime}$ is a deterministic function, $J_{t}=\mathbf{1}_{\{t \geq \gamma\}}$ jumps from 0 to 1 at the time $\gamma$ of the crash, the constant $\delta \in(0,1)$ is the relative loss of the bubble component at the time of the crash, and $T$ is the time horizon. The time of the crash $\gamma$ is a positive random variable independent ${ }^{2}$ of the Brownian motion $W$ with a distribution function $G$ that is sufficiently regular. It is assumed that $S$ is a (true) martingale, which in turn determines $\phi^{\prime}$ via $\phi^{\prime}(t)=\delta \kappa^{G}(t), t \in(0, T)$, where $\kappa^{G}=G^{\prime} /(1-G)$ is the hazard rate of $\gamma$.

A key assumption is that the hazard rate of $\gamma$ follows a log-periodic power law (LPPL)

$$
\kappa^{G}(t)=B^{\prime}|T-t|^{m-1}+C^{\prime}|T-t|^{m-1} \cos \left(\varpi \log (T-t)-\psi^{\prime}\right), \quad t \in(0, T),
$$

where $B^{\prime}, C^{\prime}, m, T, \varpi$, and $\psi^{\prime}$ are suitable real parameters; we refer to Sornette et al. (2013, Section 2.1) for interpretations. ${ }^{3}$ The JLS model confines the parameter $m$ to the interval $(0,1)$. This condition is equivalent to having a positive probability that the bubble does not burst strictly before $T$ and excludes strict local martingale dynamics for $S$ (Theorem 3.8). However, the justification for $m>0$ given in Sornette et al. (2013, Section 2.2) is debatable; see the discussion in Section 2.3. This motivates the study of a generalized JLS model.

Model class and main features. We embed the JLS model in a larger class by relaxing some of its assumptions: $G$ may be any distribution function in $C^{2}[0, T)$ with $G^{\prime}>0$ on $[0, T)$, the relative loss $\delta$ may be a $[0,1]$-valued deterministic function of time with $\delta(T)=0$, and $S$ may have a constant instantaneous expected return $\mu \in \mathbb{R}$. In particular, the probability that the bubble does not burst before or at $T$ can be chosen to be zero or positive. Instead of assuming that $S$ is a martingale, we only require that $S$ be a local martingale for $\mu=0$. The main features of this model class are:

(a) It is flexible enough to include specifications such that $S$ becomes a strict local martingale under a large class of ELMMs. This allows us to analyze to what extent the JLS model can be embedded in the strict local martingale framework.

(b) It is tractable enough to permit a semi-explicit solution to a utility maximization problem despite the incompleteness of the model class induced by the jump. This allows us to analyze how a rational agent should behave in the presence of an asset price bubble of this type.

Objective (1): The relaxed JLS model and strict local martingales. The JLS model is a martingale by definition. To meet our first objective, we thus consider the relaxed JLS model which is defined as follows: we preserve the key assumption of a log-periodic power law (1.2) for the hazard rate of the jump time but allow the parameter $m$ to be any real number (not necessarily in $(0,1)$ ), allow $\delta$ to be time-dependent in $[0,1]$ (not necessarily a constant in $(0,1)$ ), and only require $S$ to be a local martingale (not necessarily a martingale) under the physical measure. We then find that the relaxed JLS model is a strict local martingale if and only if $m \leq 0$ and the function $(1-\delta) \kappa^{G}$ is integrable on $(0, T)$ (Theorem 3.8 and Remark 3.9). In this case, the bubble bursts almost surely before $T$ and $\lim \sup _{t \uparrow \uparrow T} \delta(t)=1$, i.e., for every $\varepsilon>0$, there is a positive probability that the bubble component loses a fraction $1-\varepsilon$ of its value when the crash occurs.

\footnotetext{
${ }^{1}$ The following specification is taken from Sornette et al. (2013) (up to changes in notation); the original specification in Johansen et al. (2000) is slightly different and in particular has no explicit Brownian component.

${ }^{2}$ This assumption is not explicit in the JLS model but implicit as the postulated form of the hazard rate (1.2) does not depend on $W$.

${ }^{3}$ The parameters have to be chosen such that the hazard rate is always nonnegative; cf. v. Bothmer \& Meister (2003). This constraint was ignored in many of the early articles. The "critical time" $T>0$ is interpreted as the end of the bubble regime in Sornette et al. (2013), and the crash can happen at any time before $T$.
}

This article is protected by copyright. All rights reserved. 
Objective (2): Optimal investment. We study the problem of maximizing expected utility from terminal wealth for a power utility investor in the model class described above, assuming that the asset's instantaneous expected return is positive. ${ }^{4}$ We provide an explicit formula for the optimal strategy and the certainty equivalent of trading in the market in terms of the solution to an integral equation (or to a first-order ODE with a nonstandard terminal condition); see Theorems 4.2 and 4.7. The optimal strategy can be decomposed into two parts (Theorem 4.4): a myopic demand, which optimizes the local performance at each point in time, and a hedging demand, which takes into account how the dynamics of the asset price change globally over the investor's time frame. This decomposition allows us to conclude that investors with relative risk aversion above 1 never sell the asset short. In other words, those investors ride the bubble instead of attacking it. This theoretical insight is in line with the empirical findings of Brunnermeier \& Nagel (2004) that hedge funds were heavily invested in the stocks of the dot-com bubble despite being aware of the presence of the bubble. ${ }^{5}$

Based on numerical illustrations, we discuss the comparative statics of the optimal strategy and the certainty equivalent. Moreover, we find that the optimal strategy is not fundamentally different when the asset price process is a strict local martingale (as opposed to the situation where it is a true martingale) under a large class of ELMMs.

Default risk interpretation. Even though the underlying economic questions are completely different, from a purely mathematical perspective, the optimal investment problem could alternatively be viewed in the context of partial default risk. This problem has recently been studied by Lim \& Quenez (2011) and Jiao \& Pham (2011); there, $\gamma$ is interpreted as the time of default of the risky asset. In both articles, the optimal strategy is characterized in terms of a solution to a BSDE (with jumps). In fact, our setup can be seen as a special case of Jiao \& Pham (2011). Note, however, that our method of solving the problem (convex duality) is different from theirs (dynamic programming and BSDEs) and our solution is more explicit than theirs (in cases comparable to our setup); see Jiao \& Pham (2011, Section 4.3). More importantly, the convex duality approach to utility maximization is naturally linked to ELMMs. It is therefore better suited than dynamic programming for studying the strict local martingale property of the asset price process.

Organization of the paper. The rest of the paper is organized as follows. Section 2 fixes the probabilistic setup and notation, describes the model class, and explains how the JLS model and its relaxation are embedded therein. Section 3 contains the construction of a (sub-)class of ELMMs for our financial market and presents conditions under which the asset price is a strict local martingale under such an ELMM. The optimal investment problem is studied in Section 4. Appendix A contains a technical result that allows us to switch between certain equivalent measures and filtrations. The integral equation associated with the candidate optimal strategy is analyzed in Appendix B, while the technical aspects of the verification of its optimality are deferred to Appendix C.

\section{Model class}

Fix a finite time horizon $T>0$, and let $(\Omega, \mathcal{F}, P)$ be a probability space carrying a Brownian motion $W=\left(W_{t}\right)_{t \in[0, T]}$ and an independent random variable $\gamma$ taking values in $(0, T]$. Define the (raw) filtrations $\mathbb{F}^{W}=\left(\mathcal{F}_{t}^{W}\right)_{t \in[0, T]}, \mathbb{F}^{\gamma}=\left(\mathcal{F}_{t}^{\gamma}\right)_{t \in[0, T]}$, and $\mathbb{F}=\left(\mathcal{F}_{t}\right)_{t \in[0, T]}$ by $\mathcal{F}_{t}^{W}=\sigma\left(W_{u}: 0 \leq u \leq t\right)$, $\mathcal{F}_{t}^{\gamma}=\sigma\left(\mathbf{1}_{\{\gamma \leq u\}}: 0 \leq u \leq t\right)$, and $\mathcal{F}_{t}=\sigma\left(\mathcal{F}_{t}^{W}, \mathcal{F}_{t}^{\gamma}\right)$. Note that $\mathbb{F}^{W}$ and $\mathbb{F}^{\gamma}$ are independent under $P$ and that $\gamma$ is a stopping time with respect to $\mathbb{F}^{\gamma}$ and $\mathbb{F}$. Unless otherwise stated, all probabilistic

\footnotetext{
${ }^{4}$ Korn \& Wilmott (2002) study a related optimal investment problem where the distribution of the jump time and the jump size are unknown and the optimization follows a worst-case approach; see also Seifried (2010); Belak et al. (2014) and the references therein for this approach.

${ }^{5}$ Temin \& Voth (2004) draw the same empirical conclusion from data describing the trading activities of a well-informed bank riding the South Sea bubble in 1720 .
}

This article is protected by copyright. All rights reserved. 
notions requiring a probability measure and/or a filtration (e.g., (local) martingale properties of processes) pertain to $P$ and/or $\mathbb{F}$.

We denote the distribution function of $\gamma$ under $P$ by $G$ and assume that $G \in C^{2}[0, T)$ and $G^{\prime}>0$ on $[0, T)$; note that the law of $\gamma$ (which we denote by $\mathrm{d} G$ ) may have a point mass at $T$, in which case $\Delta G(T)>0$. We recall that the hazard rate of $\gamma$ (under $P$ ) is the function $\kappa^{G}:[0, T) \rightarrow(0, \infty)$ defined by

$$
\kappa^{G}(t)=(-\log (1-G(t)))^{\prime}=\frac{G^{\prime}(t)}{1-G(t)} .
$$

It describes the conditional probability of the jump occurring in the next instant given that the jump has not happened yet. The integrability of the hazard rate is related to the existence of a point mass of $\mathrm{d} G$ at $T$ as follows.

Proposition 2.1. The following are equivalent:

(a) The hazard rate $\kappa^{G}$ is nonintegrable on $(0, T)$.

(b) $G(T-)=1$.

(c) $\Delta G(T)=0$.

Proof. As $\gamma$ is $(0, T]$-valued, $G(T)=1$, so that the equivalence "(b) $\Leftrightarrow$ (c)" is trivial. Next, by the definition of $\kappa^{G}, \kappa^{G}(t)=-\frac{\mathrm{d}}{\mathrm{d} t} \log (1-G(t))$ for $t \in[0, T)$. Integrating both sides over $(0, T)$ yields

$$
\int_{0}^{T} \kappa^{G}(u) \mathrm{d} u=-\log (1-G(T-))
$$

(with $\log 0:=-\infty)$ and proves the equivalence "(a) $\Leftrightarrow(\mathrm{b})$ ".

\subsection{Single jump local martingales}

The asset price process in our model class is driven by the Brownian motion $W$ and a local martingale of finite variation which has a single jump at time $\gamma$. These single jump local martingales play a major role in this paper. We introduce them here and collect some of their properties; we refer to Herdegen \& Herrmann (2016) for a detailed study of the (local) martingale properties of this type of process.

For $F \in C^{1}[0, T)$, define the process $\mathcal{M}^{G} F=\left(\mathcal{M}_{t}^{G} F\right)_{t \in[0, T]}$ by

$$
\mathcal{M}_{t}^{G} F=F(t) \mathbf{1}_{\{t<\gamma\}}+\mathcal{A}^{G} F(\gamma) \mathbf{1}_{\{t \geq \gamma\}},
$$

where the function $\mathcal{A}^{G} F:[0, T] \rightarrow \mathbb{R}$ is given by

$$
\mathcal{A}^{G} F(v)= \begin{cases}F(v)-\frac{F^{\prime}(v)}{\kappa^{G}(v)}, & v \in[0, T), \\ F(v-) \mathbf{1}_{\{\Delta G(T)>0\}}, & v=T, \text { if } \lim _{t \uparrow \uparrow T} F(t) \text { exists in } \mathbb{R}, \\ 0, & v=T, \text { if } \lim _{t \uparrow \uparrow T} F(t) \text { does not exist. }\end{cases}
$$

Note that even though the function $F$ is only defined on the half-open interval $[0, T)$, the process $\mathcal{M}^{G} F$ is defined on the closed interval $[0, T]$. Each trajectory $\mathcal{M}^{G} F(\omega)$ follows the deterministic function $F$ until just before the random time $\gamma(\omega)$, has a jump at time $\gamma$ (possibly of size 0 ), and stays constant at $\mathcal{A}^{G} F(\gamma(\omega))$ from time $\gamma(\omega)$ on. The second and third lines in the definition $(2.3)$ of $\mathcal{A}^{G} F$ are only relevant if $\Delta G(T)>0$ (otherwise $\gamma<T P$-a.s.). In this case, if $\mathcal{M}^{G} F$ is a local martingale, then the left limit $F(T-)$ exists in $\mathbb{R}$ by Proposition 2.2 (b) (i) below. This has an important implication: if $\gamma=T$, then by $(2.2)-(2.3), \mathcal{M}^{G} F$ does not jump at all.

Under mild assumptions on $F$ and $G, \mathcal{M}^{G} F$ is a local $\left(P, \mathbb{F}^{\gamma}\right)$-martingale, and so by the independence of $\mathbb{F}^{W}$ and $\mathbb{F}^{\gamma}$ under $P$ also a local $(P, \mathbb{F})$-martingale. The following proposition

This article is protected by copyright. All rights reserved. 
combines several results from Herdegen \& Herrmann (2016) to provide easily checkable conditions on $F$ and $G$ for $\mathcal{M}^{G} F$ to be an integrable local martingale, a true martingale, or a square-integrable martingale with respect to the filtration $\mathbb{F}^{\gamma}$. We stress that we may apply Proposition 2.2 not only under $P$ but also under equivalent probability measures $Q \approx P$ on $(\Omega, \mathcal{F})$ as long as we replace $G$ by the distribution function of $\gamma$ under $Q$. Note, however, that unless $Q=P$, we can in general not conclude that any (local) $\left(Q, \mathbb{F}^{\gamma}\right)$-martingale is also a (local) $(Q, \mathbb{F})$-martingale. This is because $\mathbb{F}^{W}$ and $\mathbb{F}^{\gamma}$ can be dependent under $Q \neq P$. In this case, we have to resort to the technical "change of filtration lemma" (Lemma A.1 in Appendix A) which allows us to pass from $\mathbb{F}^{\gamma}$ to $\mathbb{F}$ in certain situations.

Proposition 2.2. Let $F \in C^{1}[0, T)$.

(a) The process $\mathcal{M}^{G} F$ is an integrable local $\mathbb{F}^{\gamma}$-martingale if and only if $\int_{0}^{T}\left|\mathcal{A}^{G} F(u)\right| G^{\prime}(u) \mathrm{d} u<$ $\infty$. The latter condition holds if $F$ and $\mathcal{A}^{G} F$ are bounded from below on $(0, T)$. This is automatically satisfied if $\mathcal{M}^{G} F$ is nonnegative.

(b) Suppose that $\mathcal{M}^{G} F$ is a local $\mathbb{F}^{\gamma}$-martingale.

(i) If $\Delta G(T)>0$, then $\mathcal{M}^{G} F$ is an $\mathbb{F}^{\gamma}$-martingale and the limit $\lim _{t \uparrow \uparrow T} F(t)$ exists in $\mathbb{R}$.

(ii) If $\Delta G(T)=0$ and $\mathcal{M}^{G} F$ is integrable, then $\mathcal{M}^{G} F$ is an $\mathbb{F}^{\gamma}$-martingale if and only if $\lim _{t \uparrow \uparrow T} F(t)(1-G(t))=0$.

(c) If $\int_{0}^{T}\left(\frac{F^{\prime}(u)}{\kappa^{G}(u)}\right)^{2} G^{\prime}(u) \mathrm{d} u<\infty$, then $\mathcal{M}^{G} F$ is a square-integrable $\mathbb{F}^{\gamma}$-martingale.

Proof. (a): By Lemma 3.4 in Herdegen \& Herrmann (2016), $\mathcal{M}^{G} F$ is integrable if and only if $\int_{0}^{T}\left|\mathcal{A}^{G} F(u)\right| G^{\prime}(u) \mathrm{d} u<\infty$. Moreover, in this case $\mathcal{M}^{G} F$ is automatically a local $\mathbb{F}^{\gamma}$-martingale by Lemma 3.7 in Herdegen \& Herrmann (2016). The second assertion follows from the implication "(b) $\Rightarrow$ (a)" of Lemma 2.5 in Herdegen \& Herrmann (2016).

(b): Part (i) follows from (a) and Theorem 3.5 (b)-(c) and Lemma 2.6 in Herdegen \& Herrmann (2016); part (ii) follows from Lemma 3.7 (b) in Herdegen \& Herrmann (2016).

(c): As $\int_{0}^{T} G^{\prime}(u) \mathrm{d} u=1-\Delta G(T) \leq 1, \int_{0}^{T} \frac{F^{\prime}(u)}{\kappa^{G}(u)} G^{\prime}(u) \mathrm{d} u<\infty$ by the hypothesis and Jensen's inequality. Thus, $\mathcal{M}^{G} F$ is an $H^{1}-\mathbb{F}^{\gamma}$-martingale by Lemmas 2.6, 3.4, and Theorem 3.5 in Herdegen \& Herrmann (2014) if $\Delta G(T)>0$, and by Lemmas 2.8 and 3.9 in Herdegen \& Herrmann (2014) if $\Delta G(T)=0$. Moreover, as $\mathcal{M}^{G} F$ is purely discontinuous with a single jump at $\gamma$ on $\{\gamma<T\}$, its quadratic variation satisfies

$$
\left[\mathcal{M}^{G} F\right]_{T}=\sum_{0<v \leq T}\left(\Delta \mathcal{M}^{G} F_{v}\right)^{2}=\left(\frac{F^{\prime}(\gamma)}{\kappa^{G}(\gamma)}\right)^{2} \mathbf{1}_{\{\gamma<T\}} \quad P \text {-a.s. }
$$

In particular,

$$
E\left[\left[\mathcal{M}^{G} F\right]_{T}\right]=\int_{0}^{T}\left(\frac{F^{\prime}(u)}{\kappa^{G}(u)}\right)^{2} G^{\prime}(u) \mathrm{d} u<\infty .
$$

Thus, by the Burkholder-Davis-Gundy inequality, $\mathcal{M}^{G} F$ is a square-integrable $\mathbb{F}^{\gamma}$-martingale.

\section{$2.2 \quad$ Financial market}

We consider a financial market consisting of a positive riskless asset $B=\left(B_{t}\right)_{t \in[0, T]}$, which is taken as the numéraire and without loss of generality normalized to 1 , and a risky asset $S=\left(S_{t}\right)_{t \in[0, T]}$ whose dynamics (in units of the numéraire) are given by

$$
\mathrm{d} S_{t}=S_{t-}\left(\mu \mathrm{d} t+\sigma \mathrm{d} W_{t}+\mathrm{d} \mathcal{M}^{G} \phi_{t}\right), \quad S_{0}=1 .
$$

This article is protected by copyright. All rights reserved. 
Here, $\mu \in \mathbb{R}, \sigma>0$, and $\phi \in C^{1}[0, T)$ satisfies

$$
0 \leq \phi^{\prime} \leq \kappa^{G} \text { on }[0, T) .
$$

Note that (2.5) and Proposition 2.2 (c) imply that $\mathcal{M}^{G} \phi$ is a square-integrable martingale. We may assume without loss of generality that $\phi(0)=0$. To prevent possible confusion, we stress that $S$ and $\mathcal{M}^{G} \phi$ live on the closed interval $[0, T]$ even though $\phi$ is only defined on the half-open interval $[0, T)$.

Note that the randomness in $\mathcal{M}^{G} \phi$ stems from $\gamma$, which is interpreted as the time when the bubble bursts or the crash occurs. The dynamics of the returns process $R=\left(R_{t}\right)_{t \in[0, T]}$ of $S$, defined by $R_{t}=\mu t+\sigma W_{t}+\mathcal{M}_{t}^{G} \phi$, can be summarized as follows. Prior to $\gamma, R$ consists of a drift $\left(\mu+\phi^{\prime}(t)\right) \mathrm{d} t$ and a random fluctuation $\sigma \mathrm{d} W_{t}$. Further, if $\gamma<T$, then at time $\gamma$, there is a nonpositive jump $\Delta \mathcal{M}^{G} \phi_{\gamma}=-\delta(\gamma) \mathbf{1}_{\{\gamma<T\}}$ in $R$, where $\delta:[0, T) \rightarrow[0,1]$ defined by

$$
\delta(t)=\phi(t)-\mathcal{A}^{G} \phi(t)=\frac{\phi^{\prime}(t)}{\kappa^{G}(t)}
$$

describes the absolute size of the jump of $\mathcal{M}^{G} \phi$; if $\gamma=T$, then $\mathcal{M}^{G} \phi$ does not jump (with probability 1). ${ }^{6}$ Finally, after $\gamma, R$ consists of a drift $\mu \mathrm{d} t$ and a random fluctuation $\sigma \mathrm{d} W_{t}$, i.e., it satisfies the same dynamics as the returns process of a standard Black-Scholes model. Put differently, compared to the returns process of a standard Black-Scholes model with parameters $\mu$ and $\sigma, R$ has a nonnegative extra drift $\phi^{\prime}(t) \mathrm{d} t$ prior to $\gamma$, and at time $\gamma$, there is a nonpositive jump of size $-\delta(\gamma) \mathbf{1}_{\{\gamma<T\}}$. This models - in an idealized way - a main empirical feature of a bubble, which is a strong upward trend followed by a sharp decline at bursting. For this reason, we call $\phi^{\prime}$ the instantaneous pre-crash excess return. Moreover, we call $\mu$ the instantaneous expected return. Using $\delta$, we can reformulate $(2.5)$ as

$$
0 \leq \delta \leq 1 \text { on }[0, T)
$$

which shows that the left inequality in (2.5) ensures that the instantaneous pre-crash excess return is nonnegative, whereas the right inequality ensures that the stock price is always nonnegative. If the right inequality is strict for all $t \in[0, T)$, the stock price is even positive.

\subsection{The relaxed Johansen-Ledoit-Sornette model}

Recall the dynamics of the bubble component in the JLS model from (1.1)

$$
\frac{\mathrm{d} S_{t}}{S_{t-}}=\phi^{\prime}(t) \mathrm{d} t+\sigma \mathrm{d} W_{t}-\delta \mathrm{d} J_{t},
$$

where $\phi^{\prime}(t)=\delta \kappa^{G}(t), t \in(0, T)$. Using our notation for single jump local martingales, we can combine the drift term and the jump term in (2.7) to arrive $\mathrm{at}^{7}$

$$
\frac{\mathrm{d} S_{t}}{S_{t-}}=\sigma \mathrm{d} W_{t}+\mathrm{d} \mathcal{M}^{G} \phi_{t},
$$

where $\mathcal{M}^{G} \phi$ is a single jump local martingale as introduced in (2.2) and $\phi$ is the primitive of $\phi^{\prime}$ with $\phi(0)=0$, i.e.,

$$
\phi(t)=\int_{0}^{t} \phi^{\prime}(u) \mathrm{d} u=\delta \int_{0}^{t} \kappa^{G}(u) \mathrm{d} u, \quad t \in[0, T) .
$$

We conclude that the JLS model is a special case of (2.4) with zero instantaneous expected return $\mu=0$, a hazard rate satisfying (1.2), and $\phi^{\prime}$ chosen such that $\delta(t)$, the absolute size of the jump of $\mathcal{M}^{G} \phi$ if it happens at time $t \in[0, T)$, is a constant in $(0,1)$.

\footnotetext{
${ }^{6}$ In particular, the probability that the bubble does not burst on the interval $[0, T]$ is $\Delta G(T)$, which we allow to be nonzero.

${ }^{7}$ To be precise, we assume here that $S$ evolves like a geometric Brownian motion after the crash; the JLS model does not specify what happens after the crash.
}

This article is protected by copyright. All rights reserved. 
The relaxed JLS model. We call $S$ a relaxed $J L S$ model if its dynamics are of the form (2.8) (i.e., (2.4) with $\mu=0$ ), $\phi$ satisfies (2.5), and the hazard rate $\kappa^{G}$ of $\gamma$ follows an LPPL (1.2) on $(0, T)$. The common features and differences between the JLS model and its relaxation are the following:

- The relaxed JLS model keeps the general structure of the JLS model: the returns process is composed of a time-dependent drift, a Brownian motion, and a single jump process.

- The relaxed JLS model keeps the key assumption of an LPPL for the hazard rate of the jump time.

- The relaxed JLS model does not require that $S$ be a martingale; it is, however, always a local martingale by construction.

- The relaxed JLS model does not confine the parameter $m$ to the interval $(0,1)$; instead, $m$ can be any real number.

- The relaxed JLS model does not require that the relative loss $\delta$ of $S$ at the time of the crash be a constant in $(0,1)$; instead, $\delta$ is in general a $[0,1]$-valued deterministic function of time and is determined by $\phi^{\prime}$ and $\kappa^{G}$ via (2.6).

Theorem 3.8 below implies that $m \leq 0$ is a necessary prerequisite for the relaxed JLS model to be a strict local martingale. Let us briefly discuss the restriction $m \in(0,1)$ imposed by the original JLS model.

Discussion of the restriction $m \in(0,1)$. In Sornette et al. (2013, Section 2.2), it is argued that $m$ should lie in the interval $(0,1)$. The authors state that $m<1$ is necessary to obtain an accelerating hazard rate. While this is certainly true, Brée \& Joseph (2013) point out that $m<1$ should not be an a priori restriction when fitting the LPPL (2.9) to data. A best fit with $m \geq 1$ should rather be used to reject the model.

Here, we are concerned with the restriction $m>0$. It is argued in Sornette et al. (2013) that $m>0$ is necessary to ensure that the bubble component "remains finite at all times, including $t_{c}$ [=T]" (p. 4419). However, we claim that if $m \leq 0$, then $\gamma<T P$-a.s. Indeed, if $m \leq 0$, then the hazard rate (1.2) is nonintegrable on $(0, T)$, and thus $G(T-)=1$ by Proposition 2.1 , so that $\gamma<T$ $P$-a.s. In words, the crash happens strictly before the "critical time" $T$ with probability 1 . Hence, the bubble component stays finite at all times and the argument of Sornette et al. (2013) does not justify eliminating the case $m \leq 0$ a priori. Sornette et al. (2013) also claim that the property of the JLS model that there is a positive probability that no crash occurs "makes it rational for investors to remain invested, knowing that a bubble is developing and that a crash is looming [because ...] there is a chance for investors to gain from the ramp-up of the bubble and walk away unscathed" (p. 4419). However, even if a crash happens almost surely before time $T$, it can similarly be argued that it is rational for investors to ride the bubble, knowing that the bubble will surely burst before time $T$, as long as they reduce their position before time $T$. With this strategy, they simply bet on the event that the bubble only bursts after they have closed their position. In fact, our Theorem 4.4 shows that investors with relative risk aversion larger than 1 follow such a strategy as long as the underlying asset has a positive instantaneous expected return. ${ }^{8}{ }^{8}$ We emphasize that shorting the bubble is not an arbitrage opportunity in the case where the bubble bursts almost surely before time $T$ (after all, the bubble component is a local martingale). For instance, the naive strategy of holding a (constant) short position in the bubble leads to bankruptcy with positive probability because the bubble can grow arbitrarily large if it bursts sufficiently late.

Remark 2.3. Using the formulation (2.8), we can also rigorously show that the log-periodic power law (1.2) of the hazard rate carries over to another log-periodic power law for the logarithm of the

\footnotetext{
${ }^{8}$ Investors with risk aversion less than one may also invest in the bubble under some circumstances.

${ }^{9}$ It is well known that risk-averse agents (with a finite credit line) never invest in an asset with zero instantaneous expected return.
}

This article is protected by copyright. All rights reserved. 
conditional expectation of the bubble component at some time $t \in(0, T)$ given the event that the crash has not yet happened. ${ }^{10}$ Using the independence of $\gamma$ and $W$, the conditional expectation of $S_{t}$ given that $t<\gamma$ is computed as follows

$$
\begin{aligned}
E\left[S_{t} \mid t<\gamma\right] & =\frac{1}{1-G(t)} E\left[S_{0} \mathcal{E}_{t}\left(\sigma W+\mathcal{M}^{G} \phi\right) \mathbf{1}_{\{t<\gamma\}}\right]=\frac{S_{0}}{1-G(t)} E\left[\mathcal{E}_{t}(\sigma W) \exp (\phi(t)) \mathbf{1}_{\{t<\gamma\}}\right] \\
& =S_{0} \exp (\phi(t)) .
\end{aligned}
$$

Hence, the logarithm of the expected value of the bubble component given that the crash has not happened yet reads as

$$
I(t):=\log E\left[S_{t} \mid t<\gamma\right]=\log S_{0}+\phi(t)=\log S_{0}+\delta \int_{0}^{t} \kappa^{G}(u) \mathrm{d} u .
$$

Substituting the LPPL form (1.2) of the hazard rate, using that $m \in(0,1)$, and integrating gives

$$
I(t)=A+B|T-t|^{m}+C|T-t|^{m} \cos (\varpi \log (T-t)-\psi),
$$

where $B=-\delta B^{\prime} / m, C=-\delta C^{\prime} / \sqrt{m^{2}+\varpi^{2}}$, and $A$ and $\psi$ are constants depending on $A^{\prime}, B^{\prime}, C^{\prime}$, $m, T, \varpi, \psi^{\prime}$, and $S_{0}$ (cf. equation (6) in Sornette et al. , 2013).

Equation (2.9) is at the root of the literature on log-periodic power laws in the context of financial bubbles. In 1996, Bouchaud et al. and Feigenbaum \& Freund independently suggested that the log price of a financial asset prior to a large crash can be fitted by a log-periodic power law (2.9). ${ }^{11}$ The main objective is then to obtain a prediction for the "critical time" $T$, which is interpreted as the "most probable time for the crash" (Johansen \& Sornette, 1999b) (because the hazard rate explodes at T). This approach has been widely used (see Sornette et al. (2013); Fantazzini \& Geraskin (2013) for an overview) and intensely debated in the literature (see in particular Feigenbaum, 2001b; Johansen \& Sornette, 2001; Feigenbaum, 2001a; Brée \& Joseph, 2013).

Remark 2.4. The case of $m=0$ has already been suggested by Ausloos et al. (1998a,b, 1999). They propose to replace the LPPL (2.9) by

$$
I(t)=A+B \log (T-t)+C \log (T-t) \cos (\varpi \log (T-t)-\psi) .
$$

The corresponding hazard rate

$$
\kappa^{G}(t)=B^{\prime}|T-t|^{-1}+C^{\prime}|T-t|^{-1} \cos \left(\varpi \log (T-t)-\psi^{\prime}\right)
$$

is nonintegrable on $(0, T)$, and hence $\gamma<T P$-a.s. by Proposition 2.1. To the best of our knowledge, the case $m<0$ has not been studied in the literature so far.

\section{$3 \quad$ ELMMs and the strict local martingale property}

We proceed to derive a (sub-)class of ELMMs for the financial market (2.4) and to provide conditions on the model parameters for $S$ being a strict local martingale under those ELMMs. As an application, we obtain necessary and sufficient conditions for the relaxed JLS model being a strict local martingale under the physical measure.

\footnotetext{
${ }^{10}$ See, e.g., v. Bothmer \& Meister (2003); Brée \& Joseph (2013); Sornette et al. (2013) for a formal derivation.

${ }^{11}$ We note that no distinction between the fundamental value and the bubble component was made in the early articles. Moreover, sometimes the price is fitted instead of the log price.
}

This article is protected by copyright. All rights reserved. 


\subsection{Preliminary results for single jump processes}

We first construct probability measures $Q^{\gamma} \approx P$ under which certain single jump semimartingales are square-integrable martingales. Except for the statement on square-integrability, Theorem 3.1 is essentially an application of the more general Theorem 4.2 in Herdegen \& Herrmann (2014) on the existence and characterization of ELMMs for single jump semimartingales. For the convenience of the reader and because many conditions need to be checked, we provide full details.

Theorem 3.1. Let $F, y \in C^{1}[0, T)$ be such that $0 \leq F^{\prime} \leq \kappa^{G}$ and $\inf _{t \in[0, T)} y(t)>-1$. Moreover, if $\Delta G(T)>0$, assume that

$$
\int_{0}^{T}\left|F^{\prime}(u) y(u)\right| \mathrm{d} u<\infty \quad \text { and } \quad \int_{0}^{T} \kappa^{G}(u)(1+y(u)) \mathrm{d} u<\infty .
$$

Define the functions $\zeta:[0, T) \rightarrow(0, \infty)$ and $H:[0, \infty) \rightarrow[0,1]$ by

$$
\begin{aligned}
\zeta(t) & =\exp \left(-\int_{0}^{t} \kappa^{G}(u) y(u) \mathrm{d} u\right) \\
H(t) & =1-\exp \left(-\int_{0}^{t} \kappa^{G}(u)(1+y(u)) \mathrm{d} u\right) \mathbf{1}_{\{t<T\}} .
\end{aligned}
$$

Then $\zeta$ is positive and $\mathcal{M}^{G} \zeta$ is a positive $\left(P, \mathbb{F}^{\gamma}\right)$-martingale starting at 1 . Define the measure $Q^{\gamma} \approx P$ on $\mathcal{F}_{T}^{\gamma}$ by $\frac{\mathrm{d} Q^{\gamma}}{\mathrm{d} P}=\mathcal{M}_{T}^{G} \zeta$. Then $\gamma$ has distribution function $H$ under $Q^{\gamma}$, and for $t \in[0, T)$,

$$
\begin{aligned}
\mathcal{A}^{G} \zeta(t) & =\zeta(t)(1+y(t)), \\
1-H(t) & =\zeta(t)(1-G(t)), \\
\kappa^{H}(t):=\frac{H^{\prime}(t)}{1-H(t)} & =\kappa^{G}(t)(1+y(t)) .
\end{aligned}
$$

Moreover,

$$
\mathcal{M}^{G} F+\int_{0}^{\cdot} \mathbf{1}_{\{u \leq \gamma\}} F^{\prime}(u) y(u) \mathrm{d} u=\mathcal{M}^{H}\left(\int_{0}^{\cdot} F^{\prime}(u)(1+y(u)) \mathrm{d} u\right)
$$

is a square-integrable $\left(Q^{\gamma}, \mathbb{F}^{\gamma}\right)$-martingale.

Proof. We apply the more general "removal of drift" result Theorem 4.2 in Herdegen \& Herrmann (2014). To this end, we define $A \in C^{1}[0, T)$ by $A(t)=\int_{0}^{t} F^{\prime}(u) y(u) \mathrm{d} u$ and declare that $0 / 0:=0$. Then $f:=\frac{\mathrm{d} F}{\mathrm{~d} G}=\frac{F^{\prime}}{G^{\prime}}$ and $a:=\frac{\mathrm{d} A}{\mathrm{~d} G}=f y$ on $[0, T)$. Note that if $\Delta G(T)>0$, then by $(3.1)$,

$$
\begin{aligned}
& \int_{0}^{T}|a(u)| G^{\prime}(u) \mathrm{d} u=\int_{0}^{T}|f(u) y(u)| G^{\prime}(u) \mathrm{d} u=\int_{0}^{T}\left|F^{\prime}(u) y(u)\right| \mathrm{d} u<\infty, \\
& \int_{0}^{T}|f(u)| G^{\prime}(u) \mathrm{d} u=\int_{0}^{T} F^{\prime}(u) \mathrm{d} u \leq \int_{0}^{T} \kappa^{G}(u) \mathrm{d} u=-\log \Delta G(T)<\infty,
\end{aligned}
$$

and so the assumptions in the first line of Theorem 4.2 in Herdegen \& Herrmann (2014) are satisfied. Moreover, clearly $\{f=0\} \cap(0, T) \subset\{a=0\}, \frac{a}{f}=y>-1$ on $(0, T)$ and $\int_{0}^{b}\left|\frac{a(u)}{f(u)}\right| G^{\prime}(u) \mathrm{d} u<\infty$ for each $b \in(0, T)$, i.e., the conditions (4.8)-(4.10) (and trivially also (4.11)) in Herdegen \& Herrmann (2014) are fulfilled.

We proceed to show that if $\Delta G(T)=0$, then (4.13) and (4.22) for $h=0$ in Herdegen \& Herrmann (2014) are satisfied. Indeed, the hypothesis $\inf _{t \in[0, T)} y(t)>-1$ together with the fact that $\int_{0}^{T} \kappa^{G}(u) \mathrm{d} u=\infty$ gives

$$
\int_{0}^{T}\left(\frac{a(u)}{f(u)}+1\right) \frac{G^{\prime}(u)}{1-G(u)} \mathrm{d} u=\int_{0}^{T}(1+y(u)) \kappa^{G}(u) \mathrm{d} u=\infty .
$$

This article is protected by copyright. All rights reserved. 
Next, we establish (4.14) and (4.15) in Herdegen \& Herrmann (2014) if $\Delta G(T)>0$. Set $A(T):=\int_{0}^{T} F^{\prime}(u) y(u) \mathrm{d} u$, which is well defined by (3.1). Then $\Delta A(T)=0$ and so we have (4.14) in Herdegen \& Herrmann (2014). Moreover, the identity $\frac{a}{f}=y,(3.1)$ and the identity $\int_{0}^{T} \kappa^{G}(u) \mathrm{d} u=-\log \Delta G(T)<\infty$ give

$$
\int_{0}^{T}\left|\frac{a(u)}{f(u)}\right| \frac{G^{\prime}(u)}{1-G(u)} \mathrm{d} u=\int_{0}^{T}|y(u)| \kappa^{G}(u) \mathrm{d} u<\infty .
$$

As $\frac{1}{1-G} \geq 1$ on $(0, T)$, the above yields $\int_{0}^{T}\left|\frac{a(u)}{f(u)}\right| G^{\prime}(u) \mathrm{d} u<\infty$, and we have condition (4.15) in Herdegen \& Herrmann (2014).

Now, if we define $\zeta$ by (4.16) in Herdegen \& Herrmann (2014) for $h=0$, this simplifies to (3.2), and the assertion about $\mathcal{M}^{G} \zeta$ follows from Theorem 4.2 in Herdegen \& Herrmann (2014). If we define $H$ by (3.3), then (3.5) follows from the identity $1-G(t)=\exp \left(-\int_{0}^{t} \kappa^{G}(t) \mathrm{d} t\right), t \in[0, T)$. Formula (4.23) in Herdegen \& Herrmann (2014) then shows that $\gamma$ has distribution function $H$ under $Q^{\gamma} .{ }^{12}$

Moreover, (3.4) and (3.6) are straightforward, and (3.7) follows from assertion (4.24) in Herdegen \& Herrmann (2014). Finally, note that the hypothesis $0 \leq F^{\prime} \leq \kappa^{G}$ implies via (3.6) that $0 \leq F^{\prime}(1+y) \leq \kappa^{H}$, and so Proposition 2.2 (c) (with $P$ and $G$ replaced by $Q^{\gamma}$ and $H$, respectively) yields that $\mathcal{M}^{H}\left(\int_{0}^{\cdot} F^{\prime}(u)(1+y(u)) \mathrm{d} u\right)$ is a square-integrable $\left(Q^{\gamma}, \mathbb{F}^{\gamma}\right)$-martingale.

It is decisive for our purposes to understand when the stochastic exponential of the squareintegrable $Q^{\gamma}$-martingale (3.7) is a strict local martingale under $Q^{\gamma}$. To this end, we first provide a formula for the stochastic exponential of a single jump local martingale.

Proposition 3.2. Let $F \in C^{1}[0, T)$ be such that $F(0)=0$ and $0 \leq F^{\prime} \leq \kappa^{G}\left(0 \leq F^{\prime}<\kappa^{G}\right)$. Then $\int_{0}^{T}\left|\mathcal{A}^{G}(\exp \circ F)(u)\right| G^{\prime}(u) \mathrm{d} u<\infty$ and

$$
\mathcal{E}\left(\mathcal{M}^{G} F\right)=\mathcal{M}^{G}(\exp \circ F)
$$

is a nonnegative (positive) local $\left(P, \mathbb{F}^{\gamma}\right)$-martingale.

Proof. First, note that the assumptions $0 \leq F^{\prime} \leq \kappa^{G}\left(0 \leq F^{\prime}<\kappa^{G}\right)$ imply that $\Delta \mathcal{M}^{G} F \geq-1$ $\left(\Delta \mathcal{M}^{G} F>-1\right)$. Therefore, by the formula for the stochastic exponential (see Protter, 2005, Theorem II 37), $\mathcal{E}\left(\mathcal{M}^{G} F\right)$ is nonnegative (positive). The identity (3.8) is an easy calculation. Finally, the nonnegativity of $\mathcal{M}^{G}(\exp \circ F)$ implies that $\int_{0}^{T}\left|\mathcal{A}^{G}(\exp \circ F)(u)\right| G^{\prime}(u) \mathrm{d} u<\infty$ by Proposition $2.2(\mathrm{a})$, which then also shows that $\mathcal{E}\left(\mathcal{M}^{G} F\right)$ is an integrable local $\left(P, \mathbb{F}^{\gamma}\right)$-martingale.

The next result provides a necessary and sufficient condition for $\mathcal{E}\left(\mathcal{M}^{G} F\right)$ to be a strict local $\left(P, \mathbb{F}^{\gamma}\right)$-martingale. It also shows that this strict local martingale property persists under certain changes of measure provided that the process is transformed accordingly (so that it is driftless under the new measure).

Theorem 3.3. Suppose that $\Delta G(T)=0, F(0)=0$, and $0 \leq F^{\prime}(t) \leq \kappa^{G}(t)$. Then $\mathcal{E}\left(\mathcal{M}^{G} F\right)$ is a strict local $\left(P, \mathbb{F}^{\gamma}\right)$-martingale if and only if $\int_{0}^{T}\left(\kappa^{G}(u)-F^{\prime}(u)\right) \mathrm{d} u<\infty$. Moreover, suppose that $y \in C^{1}[0, T)$ satisfies

$$
\epsilon \leq 1+y(t) \leq C+\frac{C}{F^{\prime}(t)} \mathbf{1}_{\left\{\kappa^{G}(t)<C F^{\prime}(t)\right\}}, \quad t \in[0, T)
$$

for some constants $\epsilon \in(0,1]$ and $C \geq 1$. Define $\zeta, H$, and $Q^{\gamma}$ as in Theorem 3.1. Then $\mathcal{E}\left(\mathcal{M}^{H}\left(\int_{0}^{\cdot} F^{\prime}(u)(1+y(u)) \mathrm{d} u\right)\right)$ is a strict local $\left(Q^{\gamma}, \mathbb{F}^{\gamma}\right)$-martingale if and only if $\mathcal{E}\left(\mathcal{M}^{G} F\right)$ is a strict local $\left(P, \mathbb{F}^{\gamma}\right)$-martingale.

\footnotetext{
${ }^{12}$ Note that $H$ is called $G^{Q}$ in Herdegen \& Herrmann (2014) and that $G^{Q}(T)=Q[\gamma \leq T]=P[\gamma \leq T]=1$ as $Q \approx P$.
}

This article is protected by copyright. All rights reserved. 
Proof. First, for $t \in[0, T),(2.1)$ and (3.6) give

$$
\begin{aligned}
& 1-G(t)=\exp \left(-\int_{0}^{t} \kappa^{G}(t) \mathrm{d} t\right) \\
& 1-H(t)=\exp \left(-\int_{0}^{t} \kappa^{H}(t) \mathrm{d} t\right)=\exp \left(-\int_{0}^{t} \kappa^{G}(t)(1+y(t)) \mathrm{d} t\right) .
\end{aligned}
$$

Now, the first claim follows from Propositions 3.2 and 2.2 (b) (ii) and (3.10) because

$$
(\exp \circ F)(t)(1-G(t))=\exp \left(-\int_{0}^{t}\left(\kappa^{G}(u)-F^{\prime}(u)\right) \mathrm{d} u\right), \quad t \in[0, T) .
$$

For the second claim, note that integrability of $\left(\kappa^{G}(t)-F^{\prime}(t)\right)(1+y(t))$ on $(0, T)$ is equivalent to integrability of $\kappa^{G}(t)-F^{\prime}(t)$ on $(0, T)$ as by $(3.9)$,

$$
\epsilon\left(\kappa^{G}(t)-F^{\prime}(t)\right) \leq\left(\kappa^{G}(t)-F^{\prime}(t)\right)(1+y(t)) \leq C\left(\kappa^{G}(t)-F^{\prime}(t)\right)+C(C-1) .
$$

Now, the second claim follows from the first one and Propositions 3.2 and 2.2 (b) (ii) (with $Q$ and $H$ replaced by $P$ and $G$, respectively) and (3.11) because

$$
\begin{aligned}
& \exp \left(\int_{0}^{t} F^{\prime}(u)(1+y(u)) \mathrm{d} u\right)(1-H(t)) \\
& =\exp \left(-\int_{0}^{t}\left(\kappa^{G}(u)-F^{\prime}(u)\right)(1+y(u)) \mathrm{d} u\right), \quad t \in[0, T) .
\end{aligned}
$$

\subsection{Equivalent local martingale measures}

Combining the "removal-of-drift" result Theorem 3.1 for single jump semimartingales with Girsanov's theorem for Brownian motion allows us to construct a rich subclass of ELMMs for the financial market (2.4).

Theorem 3.4. Let $y \in C^{1}[0, T)$ with $\inf _{t \in[0, T)} y(t)>-1$ be such that

$$
\int_{0}^{T}\left(\phi^{\prime}(u) y(u)\right)^{2} \mathrm{~d} u<\infty \quad \text { and } \quad \int_{0}^{T} \mathbf{1}_{\{\Delta G(T)>0\}} \kappa^{G}(u)(1+y(u)) \mathrm{d} u<\infty .
$$

Define the functions $\zeta:[0, T) \rightarrow(0, \infty)$ and $H:[0, \infty) \rightarrow[0,1]$ and the process $Z=\left(Z_{t}\right)_{t \in[0, T]}$ by

$$
\begin{aligned}
\zeta(t) & =\exp \left(-\int_{0}^{t} \kappa^{G}(u) y(u) \mathrm{d} u\right) \\
H(t) & =1-\exp \left(-\int_{0}^{t} \kappa^{G}(u)(1+y(u)) \mathrm{d} u\right) \mathbf{1}_{\{t<T\}}, \\
Z_{t} & =\mathcal{E}_{t}\left(-\int_{0}^{\cdot} \frac{1}{\sigma}\left(\mu-\phi^{\prime}(u) y(u) \mathbf{1}_{\{u \leq \gamma, u<T\}}\right) \mathrm{d} W_{u}\right) \mathcal{M}_{t}^{G} \zeta .
\end{aligned}
$$

Then $Z$ is a positive $P$-martingale starting at 1 . Define the measure $Q \approx P$ on $\mathcal{F}_{T}$ by $\frac{\mathrm{d} Q}{\mathrm{~d} P}=Z_{T}$. Then $S$ is a local $Q$-martingale and satisfies the $S D E$

$$
\mathrm{d} S_{t}=S_{t-}\left(\sigma \mathrm{d} W_{t}^{Q}+\mathrm{d} \mathcal{M}^{H}\left(\int_{0}^{\cdot} \phi^{\prime}(u)(1+y(u)) \mathrm{d} u\right)_{t}\right)
$$

where $W^{Q}=W+\int_{0}^{\cdot} \frac{1}{\sigma}\left(\mu-\phi^{\prime}(u) y(u) \mathbf{1}_{\{u \leq \gamma, u<T\}}\right) \mathrm{d} u$ is a $Q$-Brownian motion, $\gamma$ has distribution function $H$ under $Q$, and $\mathcal{M}^{H}\left(\int_{0}^{\cdot} \phi^{\prime}(u)(1+y(u)) \mathrm{d} u\right)$ is a square-integrable $Q$-martingale.

This article is protected by copyright. All rights reserved. 
Proof. For convenience, define the function $j:[0, T]^{2} \rightarrow \mathbb{R}$ by $j(t, v)=\frac{1}{\sigma}\left(\mu-\phi^{\prime}(t) y(t) \mathbf{1}_{\{t \leq v, t<T\}}\right)$ and set $Z^{1}:=\mathcal{E}\left(-\int_{0}^{*} j(u, \gamma) \mathrm{d} W_{u}\right)$ and $Z^{2}:=\mathcal{M}^{G} \zeta$.

First, $Z=Z^{1} Z^{2}$ is a $(P, \mathbb{F})$-martingale by Lemma A.1 (a) (i) with $Y^{1}=Z^{1}$ and $Y^{2}=Z^{2}$, using that $Z^{2}$ is a positive $\left(P, F F^{\gamma}\right)$-martingale by Theorem 3.1. Clearly, $Z_{0}=Z_{0}^{1}=Z_{0}^{2}=1$, and $Z^{2}$ is also a $(P, \mathbb{F})$-martingale by the independence of $\mathbb{F}^{W}$ and $\mathbb{F}^{\gamma}$ under $P$.

Second, define $Q^{1} \approx P$ on $\mathcal{F}_{T}$ by $\frac{\mathrm{d} Q^{1}}{\mathrm{~d} P}=Z_{T}^{1}$. Clearly, $Q^{1} \approx Q$ with $\frac{\mathrm{d} Q}{\mathrm{~d} Q^{1}}=Z_{T}^{2}$. By Girsanov's theorem (from $P$ to $\left.Q^{1}\right), W-\left(-\int_{0}^{*} j(u, \gamma) \mathrm{d} u\right)=W^{Q}$ is a $Q^{1}$-Brownian motion, and again by Girsanov's theorem (from $Q^{1}$ to $Q$ ) and the fact that $Z^{2}$ is purely discontinuous, $W^{Q}-\int_{0}^{\cdot} \frac{1}{Z_{u}^{2}} \mathrm{~d}\left[Z^{2}, W^{Q}\right]_{u}=W^{Q}$ is a local $Q$-martingale. By Lévy's characterization of Brownian motion, it is even a $Q$-Brownian motion.

Third, define $Q^{\gamma} \approx P$ on $\mathcal{F}_{T}$ (and on $\mathcal{F}_{T}^{\gamma}$ ) by $\frac{\mathrm{d} Q^{\gamma}}{\mathrm{d} P}=Z_{T}^{2}$. Then $\gamma$ has distribution function $H$ under $Q^{\gamma}$ by Theorem 3.1 and also under $Q$ by Lemma A.1 (b) (i), applying the latter for $X^{2, Q}=\mathbf{1}_{\{\gamma \leq t\}}$ and $s=0$.

Finally, $\mathcal{M}^{G} \phi+\int_{0}^{\cdot} \mathbf{1}_{\{u \leq \gamma\}} \phi^{\prime}(u) y(u) \mathrm{d} u=\mathcal{M}^{H}\left(\int_{0}^{\cdot} \phi^{\prime}(u)(1+y(u)) \mathrm{d} u\right)$ is a square-integrable $\left(Q^{\gamma}, \mathbb{F}^{\gamma}\right)$-martingale by Theorem 3.1 , and hence also a square-integrable $(Q, \mathbb{F})$-martingale by Lemma A.1 (b) (ii). Now, (3.16) follows from the definition of $W^{Q}$ and the dynamics of $S$ in $(2.4)$.

Note that under $Q$ as in Theorem 3.4, the stock price $S$ can be written as the product of a continuous stochastic exponential and a purely discontinuous single jump local martingale. The following technical corollary to Theorem 3.4 provides conditions under which the $Q$-martingale property of the single jump local martingale carries over to the $Q$-martingale property of the product.

Corollary 3.5. Let $y, H, Q$, and $W^{Q}$ be as in Theorem 3.4. Let $k:[0, T]^{2} \rightarrow \mathbb{R}$ be of the form $k(t, v)=k_{1}(t)+k_{2}(t) \mathbf{1}_{\{t \leq v, t<T\}}$, where $k_{1}, k_{2} \in L^{2}[0, T]$, and let $\eta \in C^{1}[0, T)$ be such that $\int_{0}^{T}\left|\mathcal{A}^{H} \eta(u)\right| H^{\prime}(u) \mathrm{d} u<\infty$. Then

$$
\mathcal{E}\left(\int_{0}^{\cdot} k(u, \gamma) \mathrm{d} W_{u}^{Q}\right) \mathcal{M}^{H} \eta
$$

is a local $Q$-martingale. It is a $Q$-martingale if and only if $\mathcal{M}^{H} \eta$ is a $Q$-martingale.

Proof. Let $j, Z, Z^{1}, Z^{2}$, and $Q^{\gamma}$ be as in the proof of Theorem 3.4. Set $\tilde{Z}^{1}:=\mathcal{E}\left(\int_{0}^{\cdot} k(u, \gamma) \mathrm{d} W_{u}^{Q}\right)$, $\tilde{Z}^{2}:=\mathcal{M}^{H} \eta, Y^{1}:=Z^{1} \tilde{Z}^{1}$, and $Y^{2}:=Z^{2} \mathcal{M}^{H} \eta$. Then $\tilde{Z}^{2}=\mathcal{M}^{H} \eta$ is a local $\left(Q^{\gamma}, \mathbb{F}^{\gamma}\right)$-martingale by Proposition 2.2 (a) (using that $\gamma$ has distribution function $H$ under $Q^{\gamma}$ ), and a short calculation gives $Y^{1}=\mathcal{E}\left(\int_{0}^{\cdot}(k-j)(u, \gamma) \mathrm{d} W_{u}\right) P$-a.s.

We have to show that $\tilde{Z}^{1} \tilde{Z}^{2}$ is a local $(Q, \mathbb{F})$-martingale, and that $\tilde{Z}^{1} \tilde{Z}^{2}$ is a $(Q, \mathbb{F})$-martingale if and only if $\tilde{Z}^{2}$ is a $(Q, \mathbb{F})$-martingale or, equivalently by Lemma A.1 (b) (ii), a $\left(Q^{\gamma}, \mathbb{F}^{\gamma}\right)$-martingale. By Bayes' theorem, it suffices to show that $Y^{1} Y^{2}=Z \tilde{Z}^{1} \tilde{Z}^{2}$ is a local $(P, \mathbb{F})$-martingale and that it is a $(P, \mathbb{F})$-martingale if and only if $Y^{2}=Z^{2} \tilde{Z}^{2}$ is a $\left(P, \mathbb{F}^{\gamma}\right)$-martingale. Recalling that $Z_{T}^{2}=\frac{\mathrm{d} Q^{\gamma}}{\mathrm{d} P}$, Bayes' theorem yields that $Y^{2}=Z^{2} \tilde{Z}^{2}$ is a local $\left(P, \mathbb{F}^{\gamma}\right)$-martingale, and Lemma A.1 (a) (ii) and (i) with $k$ replaced by $k-j$ completes the proof.

\subsection{Strict local martingale conditions}

We are now in a position to state our first main result. It gives a necessary and sufficient condition for the asset price $S$ to be a strict local martingale under certain ELMMs $Q$ constructed as in Theorem 3.4.

Theorem 3.6. Let $y$ and $Q$ be as in Theorem 3.4.

(a) If $\Delta G(T)>0$, then $S$ is always a $Q$-martingale.

This article is protected by copyright. All rights reserved. 
(b) If $\Delta G(T)=0$, assume in addition that there exist constants $\epsilon \in(0,1]$ and $C \geq 1$ such that

$$
\epsilon \leq 1+y(t) \leq C+\frac{C}{\phi^{\prime}(t)} \mathbf{1}_{\left\{\kappa^{G}(t)<C \phi^{\prime}(t)\right\}}, \quad t \in[0, T) .
$$

Then:

- $S$ is a Q-martingale if and only if $\int_{0}^{T}\left(\kappa^{G}(u)-\phi^{\prime}(u)\right) \mathrm{d} u=\infty$.

- $S$ is a strict local $Q$-martingale if and only if $\int_{0}^{T}\left(\kappa^{G}(u)-\phi^{\prime}(u)\right) \mathrm{d} u<\infty$. Moreover, in this case, $\lim \sup _{t \uparrow \uparrow T} \delta(t)=1$.

Proof. First, by $(3.16)$ and the fact that $\mathcal{M}^{H}\left(\int_{0}^{\cdot} \phi^{\prime}(u)(1+y(u)) \mathrm{d} u\right)$ is purely discontinuous,

$$
S=\mathcal{E}\left(\sigma W^{Q}\right) \mathcal{E}\left(\mathcal{M}^{H}\left(\int_{0} \phi^{\prime}(u)(1+y(u)) \mathrm{d} u\right)\right) P \text {-a.s. }
$$

Next, by Corollary 3.5, the right-hand side of (3.19) is a $Q$-martingale if and only if the second factor is. To this end, note that by Proposition 3.2, the second factor is of the form $\mathcal{M}^{H} \eta$ for $\eta=\exp \circ\left(\int_{0}^{*} \phi^{\prime}(u)(1+y(u)) \mathrm{d} u\right)$. Now, if $\Delta G(T)>0$, then $\mathcal{M}^{H} \eta$ is a $Q$-martingale by Proposition 2.2 (b) (i) (and Lemma A.1 (b) (ii) for the change of filtration). So we have (a). Otherwise, if $\Delta G(T)=0$, Theorem 3.3 (and Lemma A.1 (b) (ii) for the change of filtration) shows that $\mathcal{M}^{H} \eta$ is a $Q$-martingale if and only if $\int_{0}^{T}\left(\kappa^{G}(u)-\phi^{\prime}(u)\right) \mathrm{d} u=\infty$. This gives both equivalences in (b).

Now, suppose that $\Delta G(T)=0$ and that $\int_{0}^{T}\left(\kappa^{G}(u)-\phi^{\prime}(u)\right) \mathrm{d} u<\infty$. It remains to show that

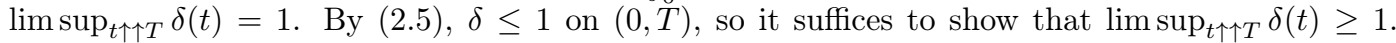

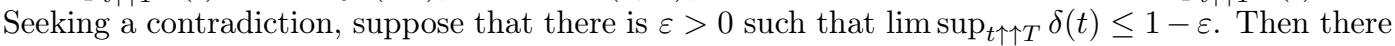
is $t_{0} \in(0, T)$ such that $\frac{\phi^{\prime}(t)}{\kappa^{G}(t)}=\delta(t) \leq 1-\varepsilon, t \in\left(t_{0}, T\right)$, or, equivalently,

$$
\kappa^{G}(t)-\phi^{\prime}(t) \geq \varepsilon \kappa^{G}(t), \quad t \in\left(t_{0}, T\right) .
$$

Recall that $\kappa^{G}$ is nonintegrable on $(0, T)$ by Proposition 2.1. As $\kappa^{G}$ is continuous on $[0, T)$, it is also nonintegrable on $\left(t_{0}, T\right)$. But then by $(3.20)$, also $\kappa^{G}-\phi^{\prime}$ is nonintegrable on $(0, T)$. This is a contradiction.

We illustrate Theorem 3.6 by giving an example where $S$ is a strict local $Q$-martingale.

Example 3.7. Let $\gamma$ be uniformly distributed on $[0,1]$, i.e., $T=1, G(t)=t$ on $[0,1]$, and let $\phi \in C^{1}[0,1)$ be given by $\phi(t)=-\log (1-t)-t$. Then $\phi^{\prime}(t)=\frac{1}{1-t}-1=\kappa^{G}(t)-1$ fulfills assumption (2.5), and so by Theorem 3.6, $S$ is a strict local martingale under any ELMM $Q$ corresponding to some $y \in C^{1}[0, T)$ with $\inf _{t \in[0, T)} y(t)>-1$ and satisfying (3.12) and (3.18) (e.g., $\left.y \equiv 0\right)$. Note that $\delta(t)=t$, the relative size of the jump of $S$ if it happens at time $t \in[0,1)$, increases linearly from 0 to 1: the later the bubble bursts, the larger the relative jump size at bursting.

\subsection{Strict local martingale characterization of the relaxed JLS model}

As an application of our first main result, Theorem 3.6, we now provide necessary and sufficient conditions for the relaxed JLS model to be a strict local martingale under the physical measure. In that case, the relative jump size $\delta(t)$ must essentially converge to 1 as $t \uparrow \uparrow T$. In other words, for each $\varepsilon>0$, there is a positive probability that the bubble component loses a fraction $1-\varepsilon$ of its value at the time of the crash.

Theorem 3.8. Suppose that the hazard rate $\kappa^{G}$ is of the form

$$
\kappa^{G}(t)=B^{\prime}|T-t|^{m-1}+C^{\prime}|T-t|^{m-1} \cos \left(\varpi \log (T-t)-\psi^{\prime}\right), \quad t \in[0, T),
$$

This article is protected by copyright. All rights reserved. 
for real parameters $B^{\prime}, C^{\prime}, m, \varpi$, and $\psi^{\prime}$ with $\left|C^{\prime}\right|<B^{\prime}$ (so that $\kappa^{G}>0$ on $[0, T)$ ). Then $S$ is a strict local martingale if and only if

$$
m \leq 0 \quad \text { and } \quad \int_{0}^{T}\left(\kappa^{G}(u)-\phi^{\prime}(u)\right) \mathrm{d} u<\infty .
$$

Moreover, in this case, $\lim \sup _{t \uparrow \uparrow T} \delta(t)=1$.

Proof. In view of the form (3.21) for $\kappa^{G}$ and the property $\left|C^{\prime}\right|<B^{\prime}$, we first note that $m \leq 0$ is equivalent to $\kappa^{G}$ being nonintegrable on $(0, T)$. This together with Proposition 2.1 shows that $m \leq 0$ if and only if $\Delta G(T)=0$. Now, the assertions follow from Theorem 3.6 (with $y \equiv 0$ ).

Remark 3.9. Recalling from (2.6) that $\delta=\frac{\phi^{\prime}}{\kappa^{G}}$, the second condition in (3.22) can alternatively be formulated as $(1-\delta) \kappa^{G}$ being integrable on $(0, T)$.

\section{Optimal investment}

Throughout this section, we assume that $\mu>0$ and $\phi \in C^{2}[0, T)$. We now analyze how a rational investor should act in the presence of an asset price bubble of the type described in Section 2.2. The optimal investment problem for a small investor is introduced in Section 4.1. The optimal strategy and the associated integral equation are heuristically derived in Section 4.2. Section 4.3 contains the corresponding rigorous existence and uniqueness result. A decomposition of the optimal strategy into its myopic and hedging demands as well as its economic interpretations are provided in Section 4.4. The certainty equivalent of trading in this market is computed in Section 4.5. Finally, Section 4.6 presents numerical illustrations of the optimal strategy and the certainty equivalent.

\subsection{Problem formulation}

We consider a small investor with initial capital $x>0$, who can trade in the financial market described in Section 2.2. For any $\mathbb{F}$-predictable, real-valued process $\pi=\left(\pi_{t}\right)_{t \in[0, T]}$ which is integrable with respect to the returns process $R$, let $X^{\pi}=\left(X_{t}^{\pi}\right)_{t \in[0, T]}$ be the unique solution to the SDE

$$
\frac{\mathrm{d} X_{t}^{\pi}}{X_{t-}^{\pi}}=\pi_{t} \frac{\mathrm{d} S_{t}}{S_{t-}}=\pi_{t} \mathrm{~d} R_{t}, \quad X_{0}^{\pi}=x .
$$

We call $\pi$ an admissible strategy if $X^{\pi}$ is positive. In this case, we can interpret $X^{\pi}$ as the wealth process corresponding to a self-financing strategy for the market $(B, S)$ (with initial capital $x$ ) and $\pi_{t}$ as the fraction of wealth invested in the stock at time $t$. We assume that the investor has a constant relative risk aversion with parameter $p>0$. The corresponding utility function is given by

$$
U(x)=\left\{\begin{array}{ll}
\frac{1}{1-p} x^{1-p} & \text { if } p \neq 1, \\
\log x & \text { if } p=1,
\end{array} \quad x>0 .\right.
$$

The investor's goal is to maximize the expected utility $E\left[U\left(X_{T}^{\pi}\right)\right]$ over all admissible strategies $\pi$ :

$$
E\left[U\left(X_{T}^{\pi}\right)\right] \rightarrow \max _{\pi}
$$

We use the method of convex duality both for the derivation and for the verification of the optimal strategy. Instead of the very deep general result of Kramkov \& Schachermayer (1999) for general incomplete semimartingale models, we only use the following well-known elementary result giving a sufficient condition for optimality; cf. the remark after Lemma 2.4 in Kallsen (2000).

This article is protected by copyright. All rights reserved. 
Proposition 4.1. Let $\hat{\pi}=\left(\hat{\pi}_{t}\right)_{t \in[0, T]}$ be an admissible strategy, $\hat{Q}$ an equivalent local martingale measure (ELMM), and $\hat{z}>0$. If

$$
(\mathrm{OC} 1) \quad U^{\prime}\left(X_{T}^{\hat{\kappa}}\right)=\hat{z} \frac{\mathrm{d} \hat{Q}}{\mathrm{~d} P} \quad \text { and } \quad(\mathrm{OC} 2) \quad E^{\hat{Q}}\left[X_{T}^{\hat{\pi}}\right]=x
$$

then $\hat{\pi}$ maximizes the expected utility $E\left[U\left(X_{T}^{\pi}\right)\right]$ over all admissible strategies $\pi$.

The ELMM $\hat{Q}$ appearing in the above result is also called the dual minimizer corresponding to the problem (4.2).

\subsection{Heuristic derivation of the optimal strategy}

We proceed to derive heuristically a candidate optimal strategy $\pi$ for the investment problem (4.2). By virtue of Proposition 4.1, we assume that a triplet $(\pi, Q, z)$ consisting of an admissible strategy $\pi$, an ELMM $Q$ for $S$ belonging to the class considered in Theorem 3.4, and a number $z>0$ satisfies the first optimality condition ${ }^{13}$

$$
\text { (OC1) } \quad U^{\prime}\left(X_{T}^{\pi}\right)=z \frac{\mathrm{d} Q}{\mathrm{~d} P} .
$$

We proceed in three steps; for ease of reading, we often drop arguments (in particular time) and do not carry out the tedious but otherwise straightforward calculations.

Step 1. As $Q$ belongs to the class of ELMMs considered in Theorem 3.4, there exists a nice function $y \in C^{1}[0, T)$ such that the density process $Z$ of $Q$ with respect to $P$ is given by

$$
Z=\mathcal{E}\left(-\int_{0}^{\cdot} \frac{1}{\sigma}\left(\mu-\phi^{\prime} y 1_{\{u \leq \gamma, u<T\}}\right) \mathrm{d} W_{u}\right) \mathcal{M}^{G} \zeta
$$

where $\zeta(t)=\exp \left(-\int_{0}^{t} \kappa^{G} y \mathrm{~d} u\right)$. By (OC1), $X_{T}^{\pi}=\left(U^{\prime}\right)^{-1}\left(z Z_{T}\right)$, and so by (4.3), after some algebra,

$$
X_{T}^{\pi}=x \mathcal{E}_{T}\left(\int_{0}^{\cdot} \frac{1}{p \sigma}\left(\mu-\phi^{\prime} y \mathbf{1}_{\{u \leq \gamma, u<T\}}\right) \mathrm{d} W_{u}^{Q}\right) \times J_{0} J(\gamma),
$$

where $J_{0}:=\frac{1}{x} z^{-\frac{1}{p}} \exp \left(\frac{1-p}{2 p^{2} \sigma^{2}} \mu^{2} T\right)$ and the function $J:[0, T] \rightarrow(0, \infty)$ is defined by

$$
J(v)= \begin{cases}\exp \left(\int_{0}^{v} \frac{1-p}{2 p^{2} \sigma^{2}} \phi^{\prime} y\left(\phi^{\prime} y-2 \mu\right)+\frac{1}{p} \kappa^{G} y \mathrm{~d} u\right)(1+y(v))^{-\frac{1}{p}} & \text { if } v<T, \\ \exp \left(\int_{0}^{T} \frac{1-p}{2 p^{2} \sigma^{2}} \phi^{\prime} y\left(\phi^{\prime} y-2 \mu\right)+\frac{1}{p} \kappa^{G} y \mathrm{~d} u\right) & \text { if } v=T .\end{cases}
$$

Step 2. By the $\operatorname{SDE}$ (4.1) for the wealth process $X^{\pi}$ and the dynamics (3.16) of $S$ under $Q$,

$$
X_{T}^{\pi}=x \mathcal{E}_{T}\left(\int_{0}^{\cdot} \pi_{u} \sigma \mathrm{d} W_{u}^{Q}\right) \times \mathcal{E}_{T}\left(\int_{0}^{\cdot} \pi_{u} \mathrm{~d} \mathcal{M}_{u}^{H}\left(\int_{0}^{\cdot} \phi^{\prime}(1+y) \mathrm{d} v\right)\right),
$$

where $H$ denotes the distribution function of $\gamma$ under $Q$. Comparing (4.4) and (4.5), we make the educated guess that the first and second factors as well as the integrands of the " $\mathrm{d} W^{Q}$-terms" coincide. In particular, a comparison of the latter gives

$$
\pi_{t}=\frac{1}{p \sigma^{2}}\left(\mu-\phi^{\prime}(t) y(t) \mathbf{1}_{\{t \leq \gamma, t<T\}}\right), \quad t \in[0, T],
$$

\footnotetext{
${ }^{13}$ Note that for the derivation of the optimal strategy we do not need to consider the second optimality condition (OC2) in Proposition 4.1. (OC2) is only needed for the verification.
}

This article is protected by copyright. All rights reserved. 
and it remains to determine the function $y$. As $\pi$ follows a deterministic function up to time $\gamma$, (a formal application of) Proposition 3.2 gives

$$
\mathcal{E}_{T}\left(\int_{0}^{\cdot} \pi_{u} \mathrm{~d} \mathcal{M}_{u}^{H}\left(\int_{0}^{\cdot} \phi^{\prime}(1+y) \mathrm{d} v\right)\right)=\mathcal{M}_{T}^{H} \xi
$$

where $\xi(t)=\exp \left(\int_{0}^{t} \frac{1}{p \sigma^{2}}\left(\mu-\phi^{\prime} y\right) \phi^{\prime}(1+y) \mathrm{d} u\right), t \in[0, T)$. Next, by (3.6) and some algebra, we obtain

$$
X_{T}^{\pi}=x \mathcal{E}_{T}\left(\int_{0}^{\cdot} \pi_{u} \sigma \mathrm{d} W_{u}^{Q}\right) \times K(\gamma)
$$

where the function $K:[0, T] \rightarrow(0, \infty)$ is defined by

$$
K(v)= \begin{cases}\exp \left(\int_{0}^{v} \frac{1}{p \sigma^{2}}\left(\mu-\phi^{\prime} y\right) \phi^{\prime}(1+y) \mathrm{d} u\right) a(v, y(v), p) & \text { if } v<T, \\ \exp \left(\int_{0}^{T} \frac{1}{p \sigma^{2}}\left(\mu-\phi^{\prime} y\right) \phi^{\prime}(1+y) \mathrm{d} u\right) & \text { if } v=T,\end{cases}
$$

and the function $a:[0, T) \times[-1, \infty) \times(0, \infty) \rightarrow \mathbb{R}$ is given by

$$
a(t, y, p)=1-\frac{1}{p \sigma^{2}} \frac{\phi^{\prime}(t)}{\kappa^{G}(t)}\left(\mu-\phi^{\prime}(t) y\right)=1-\delta(t) \frac{1}{p \sigma^{2}}\left(\mu-\phi^{\prime}(t) y\right) .
$$

Step 3. Equating the second factors on the right-hand sides of (4.4) and (4.6) gives

$$
\frac{K(v)}{J(v)}=J_{0}, \quad v \in[0, T]
$$

Using (4.8) for $v<T$ and $v=T$ and rearranging the terms yields

$$
\frac{K(v) / K(T)}{J(v) / J(T)}=1, \quad v \in[0, T) .
$$

Now, define the functions $b, m, n:[0, T) \times[-1, \infty) \times(0, \infty) \rightarrow \mathbb{R}$ by

$$
\begin{aligned}
b(t, y, p) & =\left(1+\frac{1}{p} y\right) a(t, y, p), \\
m(t, y, p) & =(1+y)^{\frac{1}{p}} a(t, y, p), \\
n(t, y, p) & =-\frac{1-p}{2 p^{2} \sigma^{2}}\left(\phi^{\prime}(t) y\right)^{2}+\kappa^{G}(t)(b(t, y, p)-1) .
\end{aligned}
$$

Then after some algebra, ${ }^{14}$

$$
\frac{K(v) / K(T)}{J(v) / J(T)}=m(v, y(v), p) \exp \left(\int_{v}^{T} n(u, y(u), p) \mathrm{d} u\right), \quad v \in[0, T) .
$$

Finally, plugging this into (4.9) and rearranging the terms shows that $y$ satisfies the integral equation

$$
m(v, y(v), p)=\exp \left(-\int_{v}^{T} n(u, y(u), p) \mathrm{d} u\right), \quad v \in[0, T) .
$$

\footnotetext{
${ }^{14}$ Note that $m$ arises from the quotient of the second factors of $K$ and $J$ and that $n$ stems from the difference of the integrands inside the exponentials of $K$ and $J$.
}

This article is protected by copyright. All rights reserved. 


\subsection{Existence and uniqueness of the optimal strategy}

We are now in a position to state our second main result. It shows that the candidate optimal strategy derived heuristically in Section 4.2 exists and is indeed optimal for the utility maximization problem (4.2).

Theorem 4.2. Fix $p \in(0, \infty)$. There exists a unique function $\hat{y} \in C^{1}[0, T)$ with $\hat{y}>-1$ satisfying the integral equation

$$
m(t, y(t), p)=\exp \left(-\int_{t}^{T} n(u, y(u), p) \mathrm{d} u\right), \quad t \in[0, T) .
$$

The strategy $\hat{\pi}=\left(\hat{\pi}_{t}\right)_{t \in[0, T]}$ defined in terms of $\hat{y}$ by

$$
\hat{\pi}_{t}=\frac{1}{p \sigma^{2}}\left(\mu-\phi^{\prime}(t) \hat{y}(t) \mathbf{1}_{\{t \leq \gamma, t<T\}}\right)
$$

is admissible and maximizes the expected utility $E\left[U\left(X_{T}^{\pi}\right)\right]$ over all admissible strategies $\pi$. Moreover, $\hat{y}$ satisfies (3.12) and (3.18).

Remark 4.3. When we speak about a solution $\hat{y}$ to the integral equation (4.13), we tacitly impose that $\int_{0}^{T}|n(u, \hat{y}(u), p)| \mathrm{d} u<\infty$. Then (4.13), the definition of $m$ in (4.11), and the requirement $\hat{y}>-1$ imply that $a(t, \hat{y}(t), p)>0, t \in[0, T)$. Economically, the latter property means that the investor's wealth is positive after the bubble has burst. Indeed, on $\{\gamma=t\}$, as the stock loses a fraction $\delta(t)$ of its value at time $t$, the wealth at time $t$ is given by

$$
\left(1-\hat{\pi}_{t}\right) X_{t-}^{\hat{\pi}}+\hat{\pi}_{t} X_{t-}^{\hat{\pi}}(1-\delta(t))=X_{t-}^{\hat{\pi}}\left(1-\delta(t) \hat{\pi}_{t}\right)=X_{t-}^{\hat{\pi}} a(t, \hat{y}(t), p)
$$

Proof of Theorem 4.2. The idea is to construct a triplet $(\hat{\pi}, \hat{Q}, \hat{z})$ which satisfies the assumptions of Proposition 4.1 and thereby yields an optimizer for the investment problem (4.2). We proceed in three steps: first, we construct a (unique) solution to the integral equation (4.13); second, we construct a triplet $(\hat{\pi}, \hat{Q}, \hat{z})$; third, we verify that this triplet satisfies the conditions (OC1) and (OC2) of Proposition 4.1.

Step 1. Theorem B.5 shows in full detail that (4.13) has a unique solution $\hat{y}>-1$ satisfying (3.12) and (3.18). Here, we only outline the main difficulties and ideas. By taking logarithms on both sides, differentiating with respect to $t$ and rearranging the terms, the integral equation (4.13) is easily transformed into an ODE of the form

$$
y^{\prime}(t)=f(t, y(t)), \quad t \in[0, T) .
$$

It is important to note that as (4.13) need not be defined for $t=T$, also $f$ need not be defined for $t=T$. However, formally letting $t \uparrow \uparrow T$ in (4.13), we find the "terminal condition"

$$
\lim _{t \uparrow \uparrow T} m(t, y(t), p)=1 .
$$

The fact that this "terminal condition" both is implicit and can only be expressed as a limit renders the ODE nonstandard. Proving existence of a solution $\hat{y}$ to the ODE (4.15) can, however, be reduced to finding a pair $\left(y^{*}, y_{*}\right)$ of so-called backward upper and backward lower solutions to (4.15) (cf. Lemma B.2). The construction of suitable $y^{*}$ and $y_{*}$ so that the solution $\hat{y}$ also satisfies (4.16) is the main technical difficulty of this first step of the proof.

Step 2. Now, we construct a triplet $(\hat{\pi}, \hat{Q}, \hat{z})$ as follows. First, by Step $1, \hat{y}$ satisfies the assumptions of Theorem 3.4 (note that (3.18) implies that $\inf _{t \in[0, T)} \hat{y}(t)>-1$ ), which yields an explicit ELMM $\hat{Q}$ for $S$. Second, we have to check that $\hat{\pi}$ defined in (4.14) is integrable with respect to the returns process $R$ and that it is admissible. The first assertion is clear from the fact that $\hat{y}$ satisfies (3.12). For the second assertion, Lemma C.1 identifies the wealth process $X^{\hat{\pi}}$ in

This article is protected by copyright. All rights reserved. 
terms of $\hat{y}$ and shows that it remains positive; the proof is mainly computational. Third, define $\hat{z}>0$ via

$$
\hat{z}^{-\frac{1}{p}}=x m(0, \hat{y}(0), p) \exp \left(-(1-p) \frac{\mu^{2}}{2 p^{2} \sigma^{2}} T\right) ;
$$

note that $m(0, \hat{y}(0), p)>0$ as $\hat{y}$ solves $(4.13)$.

Step 3. The verifications of (OC1) and (OC2) are carried out in Lemmas C.2 and C.3, respectively. The major difficulty of this step of the proof is to show that the candidate wealth process $X^{\hat{\pi}}$ is a $\hat{Q}$-martingale (i.e., $\left.(\mathrm{OC} 2)\right)$. The proof of (OC1) is mainly computational.

\subsection{Myopic and hedging demands of the optimal strategy}

A frequent goal in the context of optimal investment problems is to understand the qualitative behavior of the optimal strategy. To this end, optimal strategies are often decomposed into the sum of a myopic demand and a hedging demand; see, e.g., Adler \& Detemple (1988, Section III), Kim \& Omberg (1996, Equation (19)), Chacko \& Viceira (2005, Equation (14)), Liu (2007, Corollary 3). In discrete time, the myopic demand is the optimal strategy of an investor who treats each period as if it were the last, irrespective of the conditional distribution of any future returns (cf. Mossin, 1968). In a continuous-time setting, the myopic demand at time $t$ can be defined as the limit (if it exists) of the optimal strategy when the investment horizon $T-t$ goes to zero. One can show that in our setting, this corresponds to letting $T \downarrow t$ in the integral equation (4.13) (as one would expect formally). So the solution to the limiting equation (4.18) below can be used to define the myopic demand via (4.19). Then the hedging demand is defined as the difference between the optimal strategy and the myopic demand (cf. (4.20)). The following theorem states interesting consequences of this decomposition.

Theorem 4.4. Fix $p \in(0, \infty)$. There exists a unique function $y^{\mathrm{m}} \in C^{1}[0, T)$ with $y^{\mathrm{m}} \geq 0$ satisfying the equation

$$
m\left(t, y^{\mathrm{m}}(t), p\right)=1
$$

Let $\hat{y}$ be as in Theorem 4.2. The processes $\pi^{\mathrm{m}}=\left(\pi_{t}^{\mathrm{m}}\right)_{t \in[0, T]}$ and $\pi^{\mathrm{h}}=\left(\pi_{t}^{\mathrm{h}}\right)_{t \in[0, T]}$ defined in terms of $y^{\mathrm{m}}$ and $\hat{y}$ by

$$
\begin{aligned}
\pi_{t}^{\mathrm{m}} & =\frac{1}{p \sigma^{2}}\left(\mu-\phi^{\prime}(t) y^{\mathrm{m}}(t) \mathbf{1}_{\{t \leq \gamma, t<T\}}\right), \\
\pi_{t}^{\mathrm{h}}=\hat{\pi}_{t}-\pi_{t}^{\mathrm{m}} & =\frac{1}{p \sigma^{2}} \phi^{\prime}(t)\left(y^{\mathrm{m}}(t)-\hat{y}(t)\right) \mathbf{1}_{\{t \leq \gamma, t<T\}}
\end{aligned}
$$

are called the myopic demand and the hedging demand of the optimal strategy $\hat{\pi}$.

(a) The myopic demand satisfies

$$
0<\pi^{\mathrm{m}} \leq \frac{\mu}{p \sigma^{2}}
$$

where on $\{t \leq \gamma, t<T\}$, the right inequality is an equality if and only if $\phi^{\prime}(t)=0$.

(b) The hedging demand satisfies

$$
\pi^{\mathrm{h}} \leq 0 \text { for } p \in(0,1), \quad \pi^{\mathrm{h}}=0 \text { for } p=1, \quad \text { and } \quad \pi^{\mathrm{h}} \geq 0 \text { for } p>1 .
$$

Moreover, if $\lim \sup _{t \uparrow \uparrow T} G^{\prime}(t)<\infty$, then $\lim _{t \uparrow \uparrow T} \pi_{t}^{h}=0$ P-a.s.

Proof. The existence and uniqueness of $y^{\mathrm{m}}$ follow from Lemma B.3. To establish $y^{\mathrm{m}} \geq 0$, fix $t \in[0, T)$. By the definitions of $m$ and $a$ in (4.11) and (4.7),

$$
\begin{aligned}
1 & =m\left(t, y^{\mathrm{m}}(t), p\right)=\left(1+y^{\mathrm{m}}(t)\right)^{\frac{1}{p}} a\left(t, y^{\mathrm{m}}(t), p\right) \\
& =\left(1+y^{\mathrm{m}}(t)\right)^{\frac{1}{p}}\left(1-\frac{1}{p \sigma^{2}} \frac{\phi^{\prime}(t)}{\kappa^{G}(t)}\left(\mu-\phi^{\prime}(t) y^{\mathrm{m}}(t)\right)\right) .
\end{aligned}
$$

This article is protected by copyright. All rights reserved. 
If $y^{\mathrm{m}}(t)<0$, then the right-hand side of (4.23) is strictly smaller than 1 , which is absurd. So $y^{\mathrm{m}}$ is nonnegative.

(a): Fix $t \in[0, T]$. To establish the first inequality in (4.21), it suffices to consider the case $\phi^{\prime}(t)>0$ and $y^{\mathrm{m}}(t)>0$ and $\{t \leq \gamma, t<T\}$; for otherwise the inequality is trivially satisfied as $\mu>0$. In this case, by the definitions of $a$ and $m$ in (4.7) and (4.11), the fact that $m\left(t, y^{\mathrm{m}}(t), p\right)=1$, and $p>0$, we obtain

$$
\frac{\phi^{\prime}(t)}{\kappa^{G}(t)} \pi_{t}^{\mathrm{m}}=\frac{1}{p \sigma^{2}} \frac{\phi^{\prime}(t)}{\kappa^{G}(t)}\left(\mu-\phi^{\prime}(t) y^{\mathrm{m}}(t)\right)=1-a\left(t, y^{\mathrm{m}}(t), p\right)=1-\left(1+y^{\mathrm{m}}(t)\right)^{-\frac{1}{p}}>0,
$$

and the inequality follows. The second inequality in (4.21) follows from the nonnegativity of $y^{\mathrm{m}}$. Finally, on $\{t \leq \gamma, t<T\}$, we have $\pi_{t}^{\mathrm{m}}=\frac{1}{p \sigma^{2}}\left(\mu-\phi^{\prime}(t) y^{\mathrm{m}}(t)\right)$, and this is equal to $\frac{\mu}{p \sigma^{2}}$ if and only if $\phi^{\prime}(t) y^{\mathrm{m}}(t)=0$. By the nonnegativity of $y^{\mathrm{m}}$ and (4.23), this is equivalent to $\phi^{\prime}(t)=0$.

(b): The inequalities (4.22) follow from Theorem B.5 (noting that $y^{\mathrm{m}}=y_{*}$ for $p \leq 1$ and $y^{\mathrm{m}}=y^{*}$ for $\left.p \geq 1\right)$. The second assertion is trivial if $\Delta G(T)=0$. If $\Delta G(T)>0$, Corollary B.4 shows that $\lim _{t \uparrow \uparrow T} \phi^{\prime}(t)\left(y^{*}(t)-y_{*}(t)\right)=0$, and so a fortiori $\lim _{t \uparrow \uparrow T} \phi^{\prime}(t)\left(y^{\mathrm{m}}(t)-\hat{y}(t)\right)=0$ as $y_{*} \leq \hat{y} \leq y^{*}$ on $[0, T)$ by Theorem B.5. This completes the proof.

A couple of comments are in order.

Remark 4.5. Theorem 4.2 shows that the optimal strategy $\hat{\pi}$ is generally given in terms of the solution to an integral equation (or an ODE). By contrast, to find the myopic demand of the optimal strategy, it suffices to solve an equation for each $t$.

Remark 4.6. Our interpretation of the myopic demand in continuous time suggests that the hedging demand should approach 0 at the time horizon $T$, and this holds true under a very mild technical assumption on $G$.

The economic interpretation of the behavior of the hedging demand is as follows. After the bubble has burst, the model behaves like a Black-Scholes model with instantaneous expected return $\mu$ and instantaneous continuous variance $\sigma^{2}$. Before the crash, the instantaneous expected return is still $\mu$, but the total instantaneous variance of returns exceeds $\sigma^{2}$ due to the single jump component $\mathcal{M}^{G} \phi$. Hence, any risk-averse investor will favor the Black-Scholes market over our market (indeed, the certainty equivalent of trading in our market in Theorem 4.7 below displays a discount with respect to the Black-Scholes certainty equivalent). The later the bubble bursts, the less time an investor has to invest in the Black-Scholes market. Consequently, it is favorable for the investor if the bubble bursts early and unfavorable if it bursts late or never.

Investors with high relative risk aversion $(p>1)$ hedge against a late bursting of the bubble with a nonnegative hedging demand $\pi^{\mathrm{h}}$. Indeed, in the favorable event that the bubble bursts early, they lose more money than if they had just invested myopically and profit from the (nonnegative) instantaneous pre-crash excess return $\phi^{\prime}$ only for a short time. However, in the unfavorable event that the bubble bursts late or never, they profit significantly from the (nonnegative) instantaneous pre-crash excess return $\phi^{\prime}$ by investing more than the myopic demand; this compensates them for the only small amount of time that remains to invest in the bubble-free market.

Investors with low relative risk aversion $(p<1)$ speculate on an early bursting of the bubble with a nonpositive hedging demand $\pi^{\mathrm{m}}$. Indeed, an early bursting of the bubble is favorable to them in two ways. First, as above, they can invest in the bubble-free market for a longer time period after the crash. Second, at the time of the crash, they lose less money (or even gain money in the case of a short position coming from a hedging demand that exceeds the myopic demand in absolute value) than if they had just invested myopically. However, if the bubble bursts late or never, their optimal strategy performs worse than the myopic demand, because they profit significantly less from the instantaneous pre-crash excess return $\phi^{\prime}$.

In the limiting case of logarithmic utility $(p=1)$, investors neither hedge against nor speculate on the timing of the crash; their optimal strategy equals the myopic demand, reflecting the wellknown fact that log-investors behave myopically. Moreover, the equation $m(t, y(t), 1)=1$ reduces

This article is protected by copyright. All rights reserved. 
to a quadratic equation in $y(t)$, whose unique solution with $y>-1$ is given by $\hat{y}(t)=0$ if $\phi^{\prime}(t)=0$ and

$$
\hat{y}(t)=\frac{1}{2 \phi^{\prime}(t)}\left(\mu-\phi^{\prime}(t)-\sigma^{2} \frac{\kappa(t)}{\phi^{\prime}(t)}+\sqrt{\left(\mu-\phi^{\prime}(t)-\sigma^{2} \frac{\kappa(t)}{\phi^{\prime}(t)}\right)^{2}+4 \mu \phi^{\prime}(t)}\right)
$$

if $\phi^{\prime}(t)>0$.

\subsection{Certainty equivalent}

We proceed to calculate the certainty equivalent of the optimal strategy $\hat{\pi}$.

Theorem 4.7. If $p=1$, the certainty equivalent of trading in the market is

$$
\begin{aligned}
U^{-1}\left(E\left[U\left(X_{T}^{\hat{\pi}}\right)\right]\right)= & x \exp \left(\frac{\mu^{2}}{2 \sigma^{2}} T\right) \times \exp \left(-\int_{0}^{T} \frac{\phi^{\prime}(u)^{2} \hat{y}(u)^{2}}{2 \sigma^{2}}(1-G(u)) \mathrm{d} u\right) \\
& \times \exp \left(-\int_{0}^{T}\left(\log (1+\hat{y}(u))-\frac{\hat{y}(u)}{1+\hat{y}(u)}\right) G^{\prime}(u) \mathrm{d} u\right) .
\end{aligned}
$$

If $p \neq 1$, the certainty equivalent of trading in the market is

$$
U^{-1}\left(E\left[U\left(X_{T}^{\hat{\pi}}\right)\right]\right)=x \exp \left(\frac{\mu^{2}}{2 p \sigma^{2}} T\right) \times m(0, \hat{y}(0), p)^{-\frac{p}{1-p}} .
$$

The different factors in (4.24) have a clear economic interpretation. The first is the certainty equivalent of the Merton proportion $\frac{\mu}{\sigma^{2}}$ in the Black-Scholes model. It is shown in the proof below that the product of the first and the second factor is the certainty equivalent of the strategy $\hat{\pi}$ in the Black-Scholes model, so that the second factor alone describes the relative certainty equivalent loss due to trading the strategy $\hat{\pi}$ (instead of $\frac{\mu}{\sigma^{2}}$ ) in the Black-Scholes model. Finally, the third factor expresses the certainty equivalent loss due to the presence of the single jump component $\mathcal{M}^{G} \phi$.

In the case of general power utility, the first factor in (4.25) is again the certainty equivalent of the Merton proportion $\frac{\mu}{p \sigma^{2}}$ in the Black-Scholes model, and the second one describes the combined relative certainty equivalent loss due to trading with the strategy $\hat{\pi}$ in the Black-Scholes model and due to the presence of the single jump component $\mathcal{M}^{G} \phi$.

Proof of Theorem 4.7. First, assume that $p=1$. By the definition of the wealth process and the fact that $\left(\mu t+\sigma W_{t}\right)_{t \in[0, T]}$ is a continuous semimartingale and $\mathcal{M}^{G} \phi$ a purely discontinuous martingale,

$$
X_{T}^{\pi}=x \mathcal{E}_{T}\left(\int_{0}^{\cdot} \hat{\pi}_{u} \mathrm{~d} R_{u}\right)=x \mathcal{E}_{T}\left(\int_{0}^{\cdot} \hat{\pi}_{u} \mathrm{~d}\left(\mu u+\sigma W_{u}\right)\right) \mathcal{E}_{T}\left(\int_{0}^{\cdot} \hat{\pi}_{u} \mathrm{~d} \mathcal{M}^{G} \phi_{u}\right) P \text {-a.s. }
$$

We start by computing the expected value of the logarithm of the first factor on the right-hand side of (4.26); this corresponds exactly to the utility an investor obtains from employing the strategy $\hat{\pi}$ in the standard Black-Scholes model. As $\int_{0}^{*} \sigma \hat{\pi}_{u} \mathrm{~d} W_{u}$ is a square-integrable martingale by the definition of $\hat{\pi}$ in (4.14) and (B.16), a standard calculation gives

$$
\begin{aligned}
E\left[\log \left(\mathcal{E}_{T}\left(\int_{0}^{.} \hat{\pi}_{u} \mathrm{~d}\left(\mu u+\sigma W_{u}\right)\right)\right)\right] & =E\left[\frac{1}{2 \sigma^{2}} \int_{0}^{T}\left(\mu^{2}-\phi^{\prime}(u)^{2} \hat{y}(u)^{2} \mathbf{1}_{\{u \leq \gamma, u<T\}}\right) \mathrm{d} u\right] \\
& =\frac{\mu^{2}}{2 \sigma^{2}} T-\int_{0}^{T} \frac{\phi^{\prime}(u)^{2} \hat{y}(u)^{2}}{2 \sigma^{2}}(1-G(u)) \mathrm{d} u .
\end{aligned}
$$

This article is protected by copyright. All rights reserved. 
To compute the expected value of the logarithm of the second factor, we first note that by the dynamics of $\mathcal{M}^{G} \phi$

$$
\int_{0}^{T} \hat{\pi}_{u} \mathrm{~d} \mathcal{M}^{G} \phi_{u}=\int_{0}^{\gamma} \bar{\pi}(u) \phi^{\prime}(u) \mathrm{d} u-\bar{\pi}(\gamma) \delta(\gamma) \mathbf{1}_{\{\gamma<T\}} P \text {-a.s. }
$$

where $\bar{\pi}(u):=\frac{1}{p \sigma^{2}}\left(\mu-\phi^{\prime}(u) \hat{y}(u) \mathbf{1}_{\{u<T\}}\right), u \in[0, T]$. So by the formula for the stochastic exponential,

$$
\mathcal{E}_{T}\left(\int_{0}^{\cdot} \hat{\pi}_{u} \mathrm{~d} \mathcal{M}^{G} \phi_{u}\right)=\exp \left(\int_{0}^{T} \mathbf{1}_{\{u<\gamma\}} \bar{\pi}(u) \phi^{\prime}(u) \mathrm{d} u\right)\left(1-\bar{\pi}(\gamma) \delta(\gamma) \mathbf{1}_{\{\gamma<T\}}\right) \quad P \text {-a.s. }
$$

Thus, using the definitions of $\delta, a$, and $m$ in (2.6), (4.7), and (4.11), the definition of $\bar{\pi}$, and the fact that $m(t, \hat{y}(t), 1) \equiv 1$ by $(4.18)$ (as $\hat{\pi}=\pi^{\mathrm{m}}$ for $p=1$ by Theorem $\left.4.4(\mathrm{~b})\right)$, for $v \in[0, T)$,

$$
1-\bar{\pi}(v) \delta(v)=1-\bar{\pi}(v) \frac{\phi^{\prime}(v)}{\kappa^{G}(v)}=a(v, \hat{y}(v), 1)=\frac{m(v, \hat{y}(v), 1)}{1+\hat{y}(v)}=\frac{1}{1+\hat{y}(v)} .
$$

The above together with the definition of $\kappa^{G}$ in $(2.1)$ and the fact that $\log \left(1-\bar{\pi}(\gamma) \delta(\gamma) \mathbf{1}_{\{\gamma<T\}}\right)=$ $\log (1-\bar{\pi}(\gamma) \delta(\gamma)) \mathbf{1}_{\{\gamma<T\}}$ gives

$$
\begin{aligned}
E\left[\log \left(\mathcal{E}_{T}\left(\int_{0} \hat{\pi}_{u} \mathrm{~d} \mathcal{M}^{G} \phi_{u}\right)\right)\right] & =\int_{0}^{T} \bar{\pi}(u) \phi^{\prime}(u)(1-G(u)) \mathrm{d} u+\int_{0}^{T} \log \left(\frac{1}{1+\hat{y}(u)}\right) G^{\prime}(u) \mathrm{d} u \\
& =\int_{0}^{T}\left(\bar{\pi}(u) \frac{\phi^{\prime}(u)}{\kappa^{G}(u)}+\log \left(\frac{1}{1+\hat{y}(u)}\right)\right) G^{\prime}(u) \mathrm{d} u \\
& =-\int_{0}^{T}\left(\log (1+\hat{y}(u))-\frac{\hat{y}(u)}{1+\hat{y}(u)}\right) G^{\prime}(u) \mathrm{d} u .
\end{aligned}
$$

Putting everything together establishes (4.24).

Second, assume that $p \neq 1$. Then the optimality conditions (OC1) and (OC2) and the definition of $\hat{z}$ in (4.17) yield

$$
\begin{aligned}
E\left[U\left(X_{T}^{\hat{\pi}}\right)\right] & =\frac{1}{1-p} E\left[\left(X_{T}^{\hat{\pi}}\right)^{1-p}\right]=\frac{1}{1-p} E\left[X_{T}^{\hat{\pi}} U^{\prime}\left(X_{T}^{\hat{\pi}}\right)\right] \\
& =\frac{1}{1-p} E\left[X_{T}^{\hat{\pi}} \hat{z} \frac{\mathrm{d} \hat{Q}}{\mathrm{~d} P}\right]=\hat{z} \frac{1}{1-p} E^{\hat{Q}}\left[X_{T}^{\hat{\pi}}\right]=\hat{z} \frac{1}{1-p} x \\
& =\frac{x^{1-p}}{1-p} \exp \left((1-p) \frac{\mu^{2}}{2 p \sigma^{2}} T\right) m(0, \hat{y}(0), p)^{-p}
\end{aligned}
$$

\subsection{Numerical illustrations}

In this section, we use numerical illustrations to answer the following four questions:

(1) How do shifts in the model parameters affect the optimal strategy and its myopic and hedging demands?

(2) Can the optimal strategy involve short selling or investing more than the Merton proportion?

(3) Does the optimal strategy distinguish fundamentally between whether or not the price process is a strict local martingale under the dual minimizer $\hat{Q}$ ?

(4) How big is the welfare loss of trading in our model in comparison to optimal investment in the Black-Scholes model? And how does the welfare loss depend on shifts in the model parameters?

This article is protected by copyright. All rights reserved. 
Recall that after the bubble has burst, it is optimal to keep a constant fraction of wealth $\frac{\mu}{p \sigma^{2}}$ (the Merton proportion) in the stock. We thus focus on the optimal strategy before the crash, and all plots of trading strategies show the optimal fraction of wealth invested in the stock as a function of time given that the bubble has not burst yet.

The time horizon is always $T=1$. For questions (1) and (4), we use a cut-off exponential distribution for the jump time (in particular, with positive probability, the bubble does not burst on $[0, T])$ and a constant instantaneous pre-crash excess return $\phi^{\prime}(t)=\alpha$ for different choices of $\alpha \in(0,1)$; cf. the captions of Tables 4.1, 4.2, and 4.5. To display effects corresponding to questions (2) and (3), we use other instantaneous pre-crash excess returns and/or the uniform distribution on $[0, T]$ for the jump time (under which the bubble almost surely bursts on $[0, T]$ ); cf. Tables 4.3 and 4.4 .

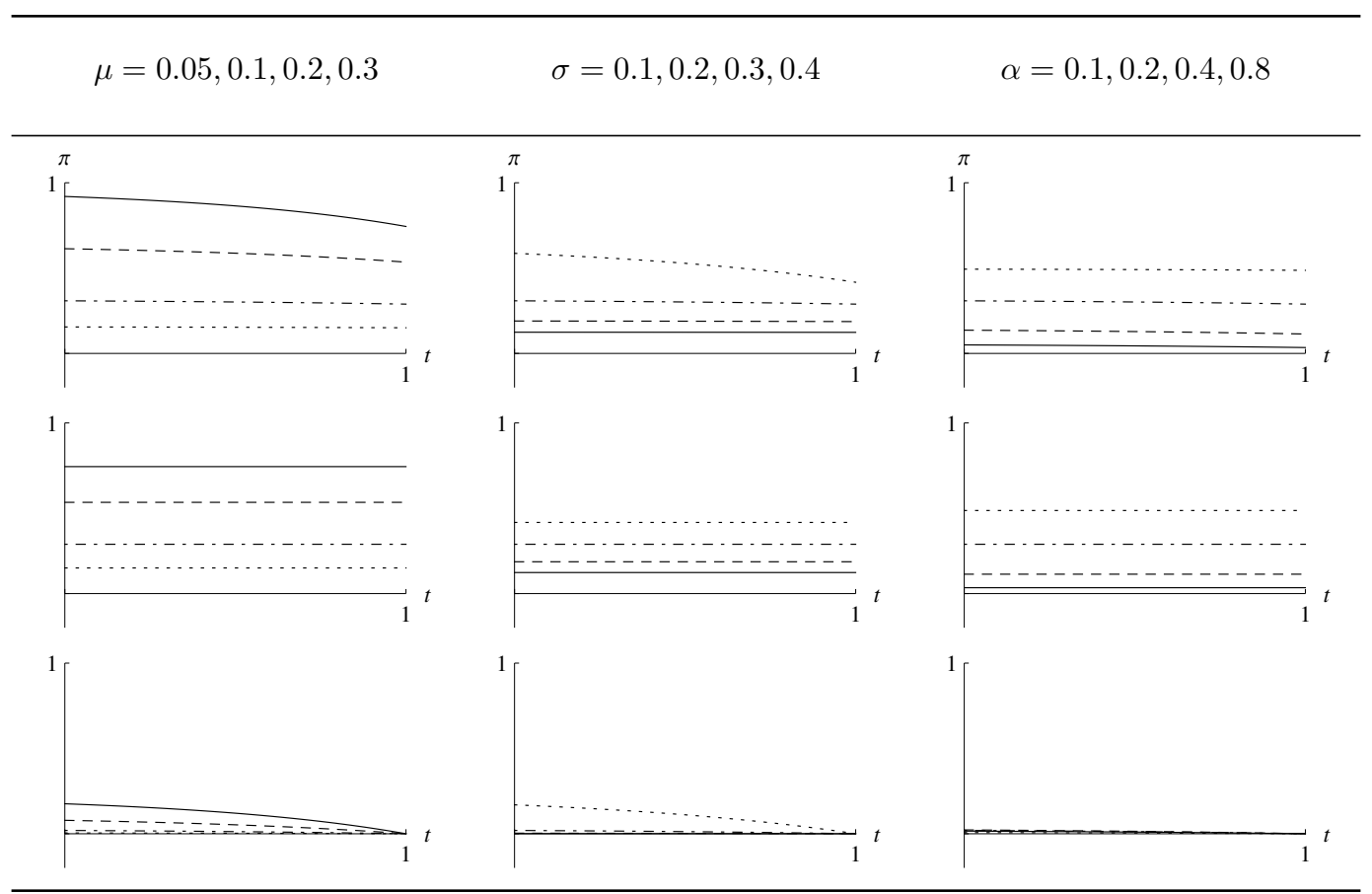

Table 4.1: Optimal strategies (top row), myopic demands (middle row) and hedging demands (bottom row) for high relative risk aversion $(p=4)$; the line strength corresponds to the size of the parameter given in the head of each column (dotted lines represent the smallest value, etc.) with default parameters $\mu=0.1, \sigma=0.2$, and $\alpha=0.2$. The setup is $T=1, G(t)=1-\exp (-t)$, and $\phi^{\prime}(t)=\alpha$; in particular, the relative jump size is $\delta(t) \equiv \alpha$.

(1) Comparative statics of the myopic and hedging demands. Theorem 4.4 states that the sign of the hedging demand $\pi^{\mathrm{h}}$ is determined by the investor's relative risk aversion $p$. Thus, we provide illustrations for the cases of high $(p>1)$ and low $(p<1)$ risk aversion. The limiting case of logarithmic utility $(p=1)$ always leads to a vanishing hedging demand and so the optimal strategy equals the myopic demand. It turns out that the qualitative behavior of the optimal strategy in this case closely resembles the behavior of the myopic demand of the optimal strategy in the case $p \neq 1$. We thus omit illustrations for the case $p=1$.

Tables 4.1 and 4.2 depict the optimal strategy before the crash as well as its decomposition into myopic and hedging demands for various choices of $\mu, \sigma$, and $\alpha$. Recall that $\alpha$ is a parameter describing the relative jump size of the stock price process $S$, for high $(p>1)$ and low $(p<1)$

This article is protected by copyright. All rights reserved. 


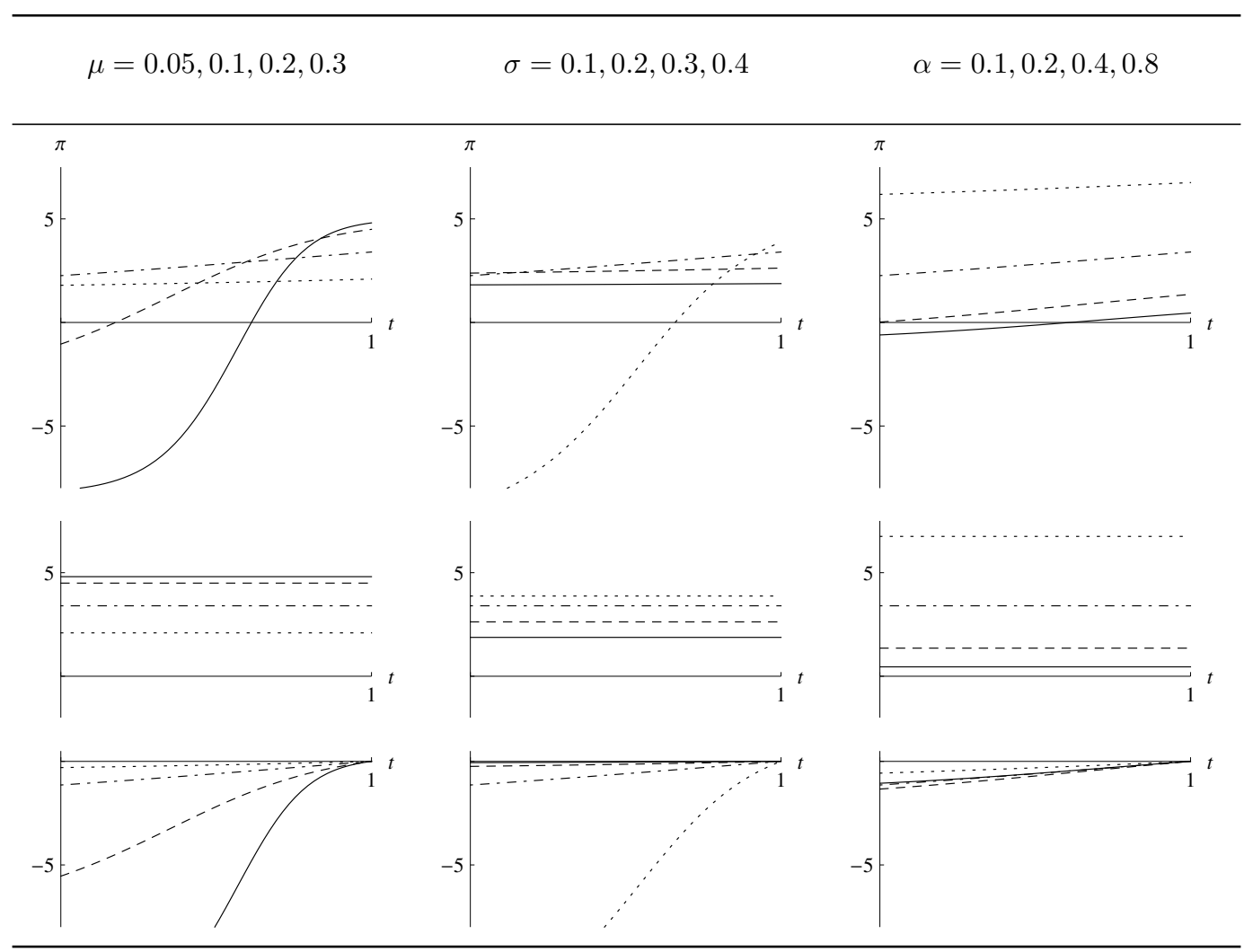

Table 4.2: Same figures as in Table 4.1 but for low relative risk aversion $(p=0.25)$.

risk aversion, respectively. The myopic demand is increasing in the instantaneous expected return $\mu$ and decreasing in the instantaneous continuous volatility $\sigma$ as well as in the relative jump size $\alpha$. Note that the myopic part is constant in Tables 4.1 and 4.2. This is because equation (4.18) determining the myopic demand is independent of time $t$ for our choice of $G$ and $\phi^{\prime}$. In general, the myopic demand need not be constant (cf. Table 4.3).

The qualitative behavior of the hedging demand, however, depends crucially on the relative risk aversion. In the case of high risk aversion $(p>1)$, the hedging demand is always nonnegative and has the same monotonicity properties as the myopic demand. In the case of low risk aversion $(p<1)$, the hedging demand is nonpositive and the monotonicity properties of the hedging demand are no longer in line with those of the myopic demand. Indeed, it is decreasing in $\mu$ (increasing in absolute value), increasing in $\sigma$ (decreasing in absolute value), and "U-shaped" in $\alpha$.

(2) Short selling and investing more than the Merton proportion. The optimal strategy includes short selling if and only if the investor's relative risk aversion $p$ is smaller than 1 and the (nonpositive) hedging demand exceeds the (nonnegative) myopic demand in absolute value; cf. the discussion after Theorem 4.4. Table 4.2 shows that short selling is amplified by "good" post-crash investment opportunities, i.e., low $\sigma$ and high $\mu$. For high relative risk aversion $(p>1)$, the myopic and hedging demands are always nonnegative (by Theorem 4.4); hence the optimal strategy never involves short selling.

When $p>1$, the optimal strategy may lie above the Merton proportion (Table 4.3). At first glance, this might be surprising as the instantaneous variance of our model is higher than in the corresponding Black-Scholes model due to the presence of the extra single jump component. However, on closer inspection, this effect can be explained by a combination of a high myopic

This article is protected by copyright. All rights reserved. 


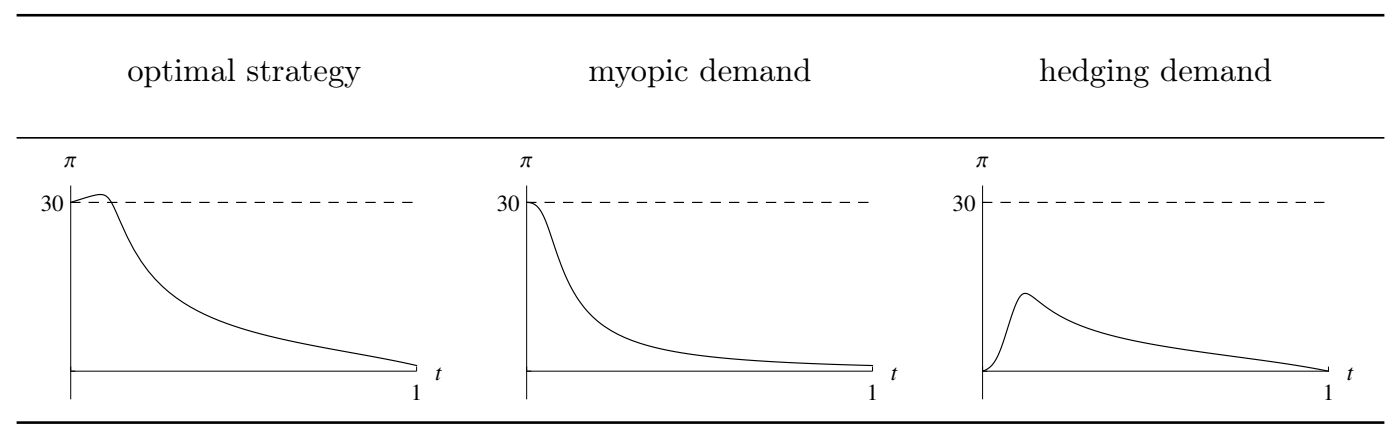

Table 4.3: Under extreme circumstances, the optimal strategy before the crash (solid, left panel) may lie above the Merton proportion (dashed). The middle and right panels show the corresponding myopic and hedging demands, respectively. The setup is $T=1, G(t)=1-\exp (-t)$, and $\phi^{\prime}(t)=$ $0.2 t$. The parameters are $\mu=0.3, \sigma=0.05$, and $p=4$.

demand at time 0 and a hedging demand that is sufficiently increasing close to time 0 .

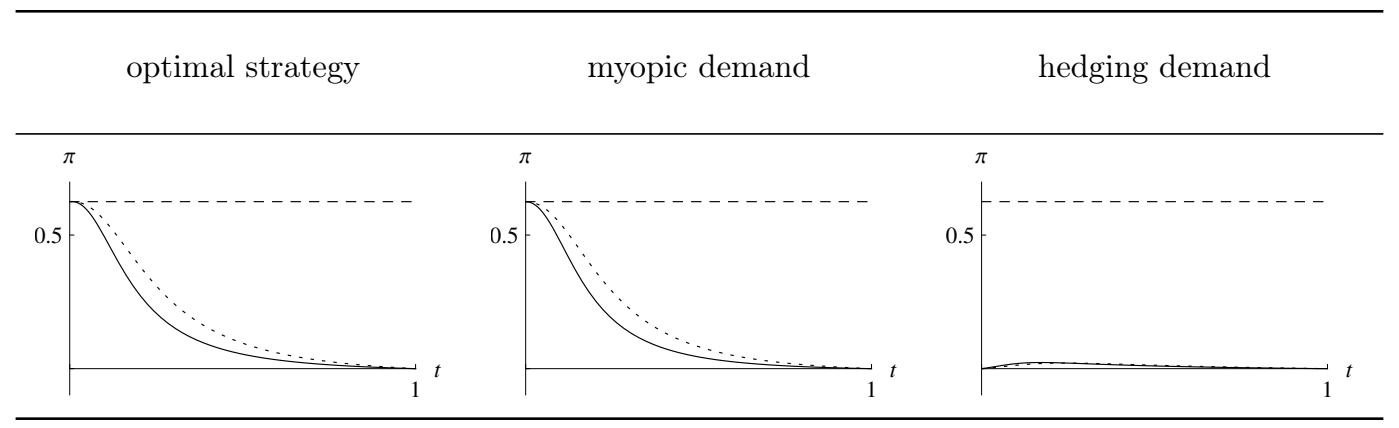

Table 4.4: The optimal strategy does not distinguish qualitatively between $S$ being a strict local martingale or a true martingale under the dual minimizer $\hat{Q}$. The setup is $T=1, G(t)=t$, and $\phi^{\prime}(t)=\alpha\left(\frac{1}{1-t}-1\right)$; in particular, the relative jump size is $\delta(t)=\alpha t$. The solid lines correspond to $\alpha=1$, for which $S$ is a strict local martingale under $\hat{Q}$, the dotted lines correspond to $\alpha=0.7$, for which $S$ is a true martingale under $\hat{Q}$. The dashed lines represent the Merton proportion. The parameters are $\mu=0.1, \sigma=0.2$, and $p=4$.

(3) Strict local martingales vs. true martingales. The investor's optimal strategy does not seem to clearly distinguish between the asset price being a strict local martingale and a true martingale under the dual minimizer $\hat{Q}$. The solid lines in Table 4.4 illustrate the optimal strategy and its decomposition into myopic and hedging demands in the case where $S$ is a strict local martingale under the dual minimizer $\hat{Q}$ (and in fact under any ELMM $Q$ obtained via Theorem 3.4 under the additional condition (3.18)). For $\alpha=1$, the setup of Table 4.4 coincides with Example 3.7. However, for any $\alpha \in[0,1)$, the stock price process $S$ is a true martingale under $\hat{Q}$ (by Theorem 3.6), and the dotted lines in Table 4.4 depict the optimal strategy and its decomposition into myopic and hedging demands for $\alpha=0.7$. The graphs show that the qualitative behavior of the optimal strategies is quite similar. In fact, the optimal strategies converge (numerically) as $\alpha \uparrow 1$.

(4) Comparative statics of the welfare loss relative to the Black-Scholes model. By Theorem 4.7, the addition of a single jump component to the Black-Scholes model reduces the certainty equivalent of trading in the market. We aim to analyze the influence of the model

This article is protected by copyright. All rights reserved. 


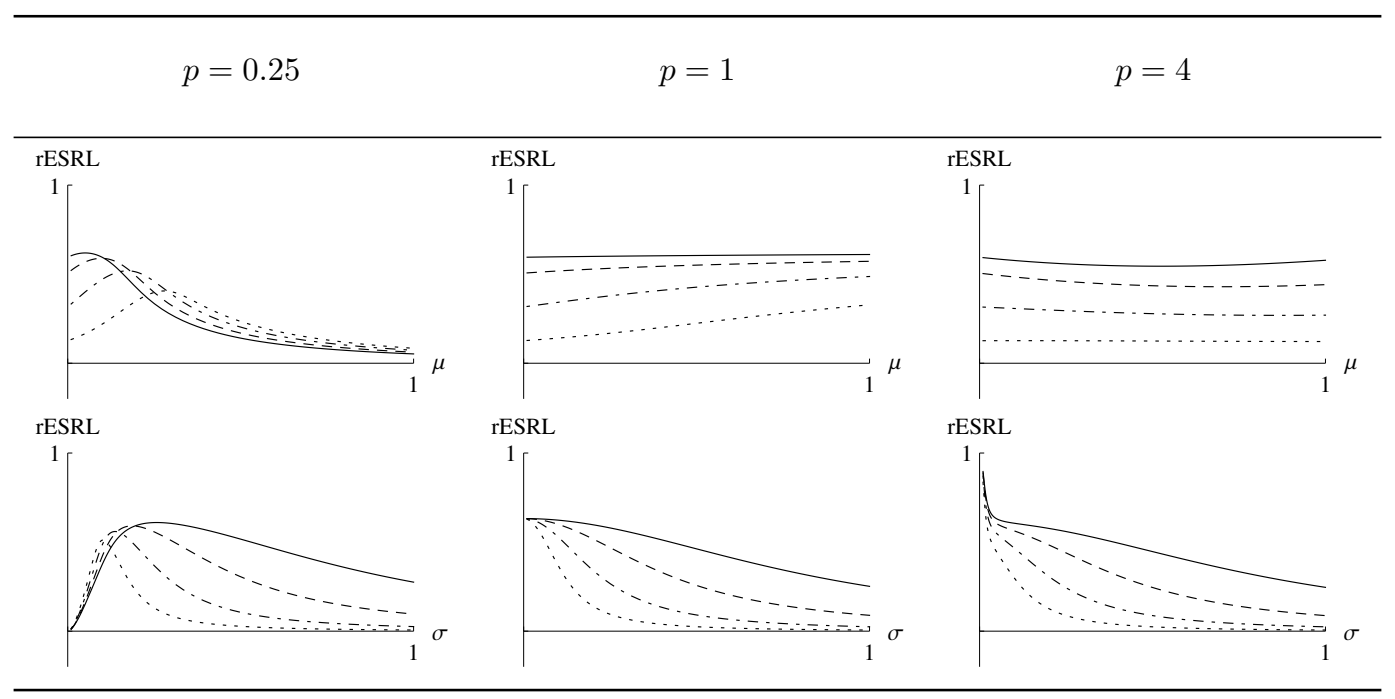

Table 4.5: Dependence of the relative equivalent safe rate loss (rESRL) on $\mu$ and $\sigma$ for $\alpha=0.1$ (dotted), $\alpha=0.2$ (dot-dashed), $\alpha=0.4$ (dashed), and $\alpha=0.8$ (solid). The setup is $T=1$, $G(t)=1-\exp (-t)$, and $\phi^{\prime}(t)=\alpha$. The parameters are $\sigma=0.2$ (top row) and $\mu=0.1$ (bottom row).

parameters on this welfare loss. A natural quantity to compare different markets is the equivalent safe rate. If $\mathrm{CE}$ denotes the certainty equivalent of trading in some market with initial capital $x$ and time horizon $T$, then the equivalent safe rate is defined as the unique solution $r:=$ ESR to the equation $x e^{r T}=\mathrm{CE}$. In other words, the investor is indifferent between trading in this market and receiving a safe annualized return $r$ on his initial capital. ${ }^{15}$

Let $\mathrm{CE}^{\mathrm{BS}}=x \exp \left(\frac{\mu^{2}}{2 p \sigma^{2}} T\right)$ denote the certainty equivalent of trading in a Black-Scholes market. The corresponding equivalent safe rate is then given by

$$
\mathrm{ESR}^{\mathrm{BS}}=\frac{1}{T} \log \left(\mathrm{CE}^{\mathrm{BS}} / x\right)=\frac{\mu^{2}}{2 p \sigma^{2}}
$$

Denoting the certainty equivalent of trading in our market given in (4.24) and (4.25) by CE, the corresponding equivalent safe rate is given by

$$
\begin{aligned}
\mathrm{ESR} & =\frac{1}{T} \log (\mathrm{CE} / x) \\
& =\mathrm{ESR}^{\mathrm{BS}}- \begin{cases}\frac{p}{1-p} \frac{1}{T} \log m(0, \hat{y}(0), p) & \text { if } p \neq 1, \\
\frac{1}{T} \int_{0}^{T}\left(\frac{\phi^{\prime}(u)^{2} \hat{y}(u)^{2}}{2 \sigma^{2} \kappa^{G}(u)}+\log (1+\hat{y}(u))-\frac{\hat{y}(u)}{1+\hat{y}(u)}\right) G^{\prime}(u) \mathrm{d} u & \text { if } p=1 .\end{cases}
\end{aligned}
$$

In order to improve the comparability over different sets of parameters, we consider the relative equivalent safe rate loss $\mathrm{rESRL}=1-\frac{\mathrm{ESR}}{\mathrm{ESR}^{\mathrm{BS}}}$ below; it is a relative measure for the incurred losses of trading in our market compared to trading in a Black-Scholes market. It follows from (4.27) that

$$
\mathrm{rESRL}=\frac{2 \sigma^{2}}{\mu^{2}} \times \begin{cases}\frac{p^{2}}{1-p} \frac{1}{T} \log m(0, \hat{y}(0), p) & \text { if } p \neq 1 \\ \frac{1}{T} \int_{0}^{T}\left(\frac{\phi^{\prime}(u)^{2} \hat{y}(u)^{2}}{2 \sigma^{2} \kappa^{G}(u)}+\log (1+\hat{y}(u))-\frac{\hat{y}(u)}{1+\hat{y}(u)}\right) G^{\prime}(u) \mathrm{d} u & \text { if } p=1\end{cases}
$$

\footnotetext{
${ }^{15}$ In a different setting, Gerhold et al. (2014) define the equivalent safe rate slightly differently: they look at the "long-run" equivalent safe rate, i.e., the limit as $T \uparrow \infty$.
}

This article is protected by copyright. All rights reserved. 
Table 4.5 illustrates the dependence of the rESRL on the model parameters $\mu$ and $\sigma$ as well as on the parameter $\alpha$ describing the relative jump size of the stock price process $S$. In the case $p=4$, the rESRL is increasing in $\alpha$ and decreasing in $\sigma$ while being almost constant in $\mu$. This is because the diffusive part becomes more and more dominant against the jump part with decreasing $\alpha$ or increasing $\sigma$, so that our model resembles more and more the Black-Scholes model.

In the case $p=0.25$, the dependencies are much less clear. On the one hand, if $\mu$ is sufficiently small and/or $\sigma$ is sufficiently large, then the rESRL is increasing in $\alpha$. The reason is that in this case, as observed above (cf. Table 4.2), the optimal strategy does not involve short selling. Therefore, the higher $\alpha$, the higher the losses when the bubble bursts, so that the investor is better off with small jump sizes; this means that the rESRL is increasing in $\alpha$.

On the other hand, if $\mu$ is high enough and/or $\sigma$ is low enough, so that the optimal strategy involves a significant short position for a significant amount of time, then the investor's wealth is likely to increase when the bubble bursts. Under these circumstances, the investor prefers larger jump sizes; in other words, the rESRL is decreasing in $\alpha$.

An interesting observation is that for small $\sigma$, i.e., when the jump part dominates the diffusive part, investors with a small relative risk aversion lose only a small fraction of their ESR compared to an investment into a Black-Scholes market. On the contrary, investors with high relative risk aversion face huge losses in their ESR for small $\sigma$. This is due to short selling opportunities for investors with low relative risk aversion; cf. the discussion after Theorem 4.4.

\section{A Change of filtration}

Recall from Section 2 that the (raw) filtrations $\mathbb{F}^{W}=\left(\mathcal{F}_{t}^{W}\right)_{t \in[0, T]}, \mathbb{F}^{\gamma}=\left(\mathcal{F}_{t}^{\gamma}\right)_{t \in[0, T]}$, and $\mathbb{F}=$ $\left(\mathcal{F}_{t}\right)_{t \in[0, T]}$ are defined by $\mathcal{F}_{t}^{W}=\sigma\left(W_{u}: 0 \leq u \leq t\right), \mathcal{F}_{t}^{\gamma}=\sigma\left(\mathbf{1}_{\{\gamma \leq u\}}: 0 \leq u \leq t\right)$, and $\mathcal{F}_{t}=$ $\sigma\left(\mathcal{F}_{t}^{W}, \mathcal{F}_{t}^{\gamma}\right)$, and that $\mathbb{F}^{W}$ and $\mathbb{F}^{\gamma}$ are independent under $P$. The key message of the following technical result is that (local) $\mathbb{F}^{\gamma}$-martingales are (local) $\mathbb{F}$-martingales not only under $P$ but also under certain equivalent measures $Q \approx P$, under which $\mathbb{F}^{W}$ and $\mathbb{F}^{\gamma}$ are no longer independent.

Lemma A.1. Let the function $k:[0, T]^{2} \rightarrow \mathbb{R}$ be of the form $k(t, v)=\hat{k}(t)+\check{k}(t) \mathbf{1}_{\{t \leq v, t<T\}}$, where $\hat{k}, \breve{k} \in L^{2}[0, T]$. Set $Y^{1}:=\mathcal{E}\left(\int_{0}^{\cdot} k(u, \gamma) \mathrm{d} W_{u}\right)$ and let $Z^{2}$ be a positive $\mathbb{F}^{\gamma}$-martingale with $Z_{0}^{2}=1$.

(a) Let $Y^{2}$ be an $\mathbb{F}^{\gamma}$-adapted càdlàg process.

(i) The following are equivalent:

$$
\begin{aligned}
& Y^{2} \text { is an } \mathbb{F}^{\gamma} \text {-martingale; } \\
& Y^{2} \text { is an } \mathbb{F} \text {-martingale; } \\
& Y^{1} Y^{2} \text { is an } \mathbb{F} \text {-martingale. }
\end{aligned}
$$

(ii) If $Y^{2}$ is a local $\mathbb{F}^{\gamma}$-martingale, then $Y^{2}$ and $Y^{1} Y^{2}$ are local $\mathbb{F}$-martingales.

(b) Define $Q^{\gamma}, Q \approx P$ on $\mathcal{F}_{T}$ by $\frac{\mathrm{d} Q^{\gamma}}{\mathrm{d} P}=Z_{T}^{2}$ and $\frac{\mathrm{d} Q}{\mathrm{~d} P}=Y_{T}^{1} Z_{T}^{2} \cdot{ }^{16}$ Let $X^{2, Q}$ be an $\mathcal{F}_{T}^{\gamma}$-measurable random variable and $Y^{2, Q}$ an $\mathbb{F}^{\gamma}$-adapted càdlàg process.

(i) $X^{2, Q}$ is $Q$-integrable if and only if it is $Q^{\gamma}$-integrable, and in this case,

$$
E^{Q}\left[X^{2, Q} \mid \mathcal{F}_{s}\right]=E^{Q^{\gamma}}\left[X^{2, Q} \mid \mathcal{F}_{s}^{\gamma}\right] \quad P \text {-a.s., } \quad s \in[0, T]
$$

(ii) $Y^{2, Q}$ is a (square-integrable) $\left(Q^{\gamma}, \mathbb{F}^{\gamma}\right)$-martingale if and only if it is a (square-integrable) $(Q, \mathbb{F})$-martingale.

(iii) If $Y^{2, Q}$ is a local $\left(Q^{\gamma}, \mathbb{F}^{\gamma}\right)$-martingale, then it is also a local $(Q, \mathbb{F})$-martingale.

\footnotetext{
${ }^{16}$ Note that (a) (i) with $Y^{2}:=Z^{2}$ shows that $Y^{1} Z^{2}$ is a positive $\mathbb{F}$-martingale with $Y_{0}^{1} Z_{0}^{2}=1$.
}

This article is protected by copyright. All rights reserved. 
Proof. First, we show that an $\mathcal{F}_{T}^{\gamma}$-measurable random variable $X^{2}$ is integrable if and only if $Y_{T}^{1} X^{2}$ is so, and in this case,

$$
E\left[Y_{T}^{1} X^{2} \mid \mathcal{F}_{s}\right]=Y_{s}^{1} E\left[X^{2} \mid \mathcal{F}_{s}^{\gamma}\right] \quad P \text {-a.s., } \quad s \in[0, T] .
$$

By linearity, we may assume that $X^{2}$ is nonnegative. Then the first assertion follows from (A.2) for $s=0$. To establish (A.2), fix $s \in[0, T]$ and set $\mathcal{C}_{s}:=\left\{C=C^{W} \cap\{\gamma \leq u\}: C^{W} \in \mathcal{F}_{s}^{W}, u \leq s\right\}$. Then $\mathcal{C}_{s}$ is an intersection-closed generator of $\mathcal{F}_{s}$, and by the $\mathcal{F}_{s}$-measurability of both sides of (A.2), the positivity of $Y_{s}^{1}$, and a monotone class argument, it suffices to show that

$$
E\left[\frac{Y_{T}^{1}}{Y_{s}^{1}} X^{2} \mathbf{1}_{C}\right]=E\left[E\left[X^{2} \mid \mathcal{F}_{s}^{\gamma}\right] \mathbf{1}_{C}\right] \quad \text { for all } C \in \mathcal{C}_{s} \cup\{\Omega\} .
$$

To establish (A.3), for fixed $v \in[0, T]$, set $Y^{1, v}=\mathcal{E}\left(\int_{0}^{\cdot} k(u, v) \mathrm{d} W_{u}\right)$. By the assumption on $k$ and Novikov's condition, each $Y^{1, v}$ is a positive $\mathbb{F}$-martingale with $Y_{0}^{1, v}=1$ and hence satisfies

$$
E\left[\frac{Y_{T}^{1, v}}{Y_{s}^{1, v}} \mathbf{1}_{A}\right]=E\left[\mathbf{1}_{A}\right], \quad s \in[0, T], A \in \mathcal{F}_{s} .
$$

Moreover, by the independence of $\mathcal{F}_{T}^{\gamma}=\sigma(\gamma)$ and $W$, a monotone class argument, and (A.4),

$$
E\left[\frac{Y_{T}^{1}}{Y_{s}^{1}} \mid \mathcal{F}_{T}^{\gamma}\right]=\left.E\left[\frac{Y_{T}^{1, v}}{Y_{s}^{1, v}}\right]\right|_{v=\gamma}=1 \quad P \text {-a.s., } \quad s \in[0, T] .
$$

Now, (A.3) for $C=\Omega$ follows from the $\mathcal{F}_{T}^{\gamma}$-measurability of $X^{2}$ and (A.5) via

$$
E\left[\frac{Y_{T}^{1}}{Y_{s}^{1}} X^{2}\right]=E\left[E\left[\frac{Y_{T}^{1}}{Y_{s}^{1}} \mid \mathcal{F}_{T}^{\gamma}\right] X^{2}\right]=E\left[X^{2}\right]=E\left[E\left[X^{2} \mid \mathcal{F}_{s}^{\gamma}\right]\right] .
$$

If $C=C^{W} \cap\{\gamma \leq u\}$, where $C^{W} \in \mathcal{F}_{s}^{W}$ and $u \leq s$, then $Y_{T}^{1} / Y_{s}^{1}=Y_{T}^{1,0} / Y_{s}^{1,0}$ on $C$ as $k(t, v)=\hat{k}(t)=k(t, 0)$ for $t>v$. Moreover, $Y_{T}^{1,0} / Y_{s}^{1,0} \mathbf{1}_{C^{W}}$ is $\mathcal{F}_{T}^{W}$-measurable and $X^{2} \mathbf{1}_{\{\gamma \leq u\}}$ and $E\left[X^{2} \mid \mathcal{F}_{t}^{\gamma}\right] \mathbf{1}_{\{\gamma \leq u\}}$ are $\mathcal{F}_{T}^{\gamma}$-measurable. This, the independence of $\mathcal{F}_{T}^{W}$ and $\mathcal{F}_{T}^{\gamma}$, and (A.4) for $A=C^{W}$ yield

$$
\begin{aligned}
E\left[\frac{Y_{T}^{1}}{Y_{s}^{1}} X^{2} \mathbf{1}_{C}\right] & =E\left[\frac{Y_{T}^{1,0}}{Y_{s}^{1,0}} X^{2} \mathbf{1}_{C}\right]=E\left[\frac{Y_{T}^{1,0}}{Y_{s}^{1,0}} \mathbf{1}_{C}\right] E\left[X^{2} \mathbf{1}_{\{\gamma \leq u\}}\right] \\
& =E\left[\mathbf{1}_{C^{W}}\right] E\left[E\left[X^{2} \mid \mathcal{F}_{s}^{\gamma}\right] \mathbf{1}_{\{\gamma \leq u\}}\right]=E\left[E\left[X^{2} \mid \mathcal{F}_{s}^{\gamma}\right] \mathbf{1}_{C^{W}} \mathbf{1}_{\{\gamma \leq u\}}\right] \\
& =E\left[E\left[X^{2} \mid \mathcal{F}_{s}^{\gamma}\right] \mathbf{1}_{C}\right] .
\end{aligned}
$$

Second, we establish (a). By the first part of the proof, $X^{2}:=Y_{T}^{2}$ is integrable if and only if $Y_{T}^{1} Y_{T}^{2}$ is so, and in this case,

$$
E\left[Y_{T}^{1} Y_{T}^{2} \mid \mathcal{F}_{s}\right]=Y_{s}^{1} E\left[Y_{T}^{2} \mid \mathcal{F}_{s}^{\gamma}\right] \quad P \text {-a.s., } \quad s \in[0, T] .
$$

As $Y_{s}^{1}>0 P$-a.s., (A.6) shows that $Y^{2}$ is an $\mathbb{F}^{\gamma}$-martingale if and only if $Y^{1} Y^{2}$ is an $\mathbb{F}$-martingale, and for $Y^{1} \equiv 1$ (i.e., for $k \equiv 0$ ), this implies that $Y^{2}$ is an $\mathbb{F}^{\gamma}$-martingale if and only if it is an $\mathbb{F}$ martingale. So we have (i). To establish (ii), let $\tau$ be a $[0, T]$-valued $\mathbb{F}^{\gamma}$ - (and a fortiori $\mathbb{F}$-)stopping time. Then by the $\mathbb{F}$-martingale property of $Y^{1}$ (which follows from Novikov's condition and the assumptions on $k$ ) and (A.2) for $X^{2}=\left|Y_{\tau}^{2}\right|$ and $s=0$,

$$
E\left[Y_{\tau}^{1}\left|Y_{\tau}^{2}\right|\right]=E\left[E\left[Y_{T}^{1} \mid \mathcal{F}_{\tau}\right]\left|Y_{\tau}^{2}\right|\right]=E\left[E\left[Y_{T}^{1}\left|Y_{\tau}^{2}\right| \mid \mathcal{F}_{\tau}\right]\right]=E\left[Y_{T}^{1}\left|Y_{\tau}^{2}\right|\right]=E\left[\left|Y_{\tau}^{2}\right|\right]
$$

This article is protected by copyright. All rights reserved. 
This implies that $Y_{\tau}^{1} Y_{\tau}^{2}$ is integrable if and only if $Y_{\tau}^{2}$ is so. Now, if the stopped process $\left(Y^{2}\right)^{\tau}$ is an $\mathbb{F}^{\gamma}$-martingale, by the $\mathbb{F}$-martingale property of $Y^{1}$, the $\mathbb{F}^{\gamma}$-martingale property of $\left(Y^{2}\right)^{\tau}$, and (A.2) for $X:=\left(Y^{2}\right)_{T}^{\tau}$, for $s \in[0, T]$,

$$
\begin{aligned}
E\left[Y_{\tau}^{1} Y_{\tau}^{2} \mathbf{1}_{\{\tau>s\}} \mid \mathcal{F}_{s}\right] & =E\left[E\left[Y_{T}^{1} \mid \mathcal{F}_{\tau \vee s}\right] Y_{\tau}^{2} \mathbf{1}_{\{\tau>s\}} \mid \mathcal{F}_{s}\right]=E\left[E\left[Y_{T}^{1} Y_{\tau}^{2} \mathbf{1}_{\{\tau>s\}} \mid \mathcal{F}_{\tau \vee s}\right] \mid \mathcal{F}_{s}\right] \\
& =E\left[Y_{T}^{1}\left(Y^{2}\right)_{T}^{\tau} \mid \mathcal{F}_{s}\right] \mathbf{1}_{\{\tau>s\}}=Y_{s}^{1} E\left[\left(Y^{2}\right)_{T}^{\tau} \mid \mathcal{F}_{s}^{\gamma}\right] \mathbf{1}_{\{\tau>s\}} \\
& =\left(Y^{1}\right)_{s}^{\tau}\left(Y^{2}\right)_{s}^{\tau} \mathbf{1}_{\{\tau>s\}} P \text {-a.s. }
\end{aligned}
$$

Thus, $\left(Y^{1}\right)^{\tau}\left(Y^{2}\right)^{\tau}$ is an $\mathbb{F}$-martingale because

$$
E\left[\left(Y^{1}\right)_{T}^{\tau}\left(Y^{2}\right)_{T}^{\tau} \mid \mathcal{F}_{s}\right]=Y_{\tau}^{1} Y_{\tau}^{2} \mathbf{1}_{\{\tau \leq s\}}+E\left[Y_{\tau}^{1} Y_{\tau}^{2} \mathbf{1}_{\{\tau>s\}} \mid \mathcal{F}_{s}\right]=\left(Y^{1}\right)_{s}^{\tau}\left(Y^{2}\right)_{s}^{\tau} \quad P \text {-a.s. }
$$

For $Y^{1} \equiv 1$ (i.e., for $k \equiv 0$ ), this also implies that $\left(Y^{2}\right)^{\tau}$ is an $\mathbb{F}$-martingale. So if $\left(\tau_{n}\right)_{n \in \mathbb{N}}$ is a localizing sequence for $Y^{2}$ in $\mathbb{F}^{\gamma}$, it is also a localizing sequence for $Y^{2}$ and $Y^{1} Y^{2}$ in $\mathbb{F}$, and we have (ii).

Finally, we establish (b). For (i), set $X^{2}=Z_{T}^{2} X^{2, Q}$. Then by Bayes' theorem, (A.2) for $s=0$, and again Bayes' theorem,

$$
E^{Q}\left[\left|X^{2, Q}\right|\right]=E^{P}\left[Y_{T}^{1} Z_{T}^{2}\left|X^{2, Q}\right|\right]=E^{P}\left[Y_{T}^{1}\left|X^{2}\right|\right]=E^{P}\left[\left|X^{2}\right|\right]=E^{P}\left[Z_{T}^{2}\left|X^{2, Q}\right|\right]=E^{Q^{\gamma}}\left[\left|X^{2, Q}\right|\right],
$$

which shows that $X^{2, Q}$ is $Q$-integrable if and only if it is $Q^{\gamma}$-integrable. Now, the same argument yields (A.1) using (A.2) for general $s \in[0, T]$.

For (ii) and (iii), set $Y^{2}=Z^{2} Y^{2, Q}$. Then by Bayes' theorem, $Y^{2, Q}$ is a (local) $\left(Q^{\gamma}, \mathbb{F}^{\gamma}\right)$ martingale if and only if $Y^{2}$ is a (local) $\left(P, \mathbb{F}^{\gamma}\right)$-martingale. Likewise by Bayes' theorem, $Y^{2, Q}$ is a (local) $(Q, \mathbb{F})$-martingale if and only if $Y^{1} Y^{2}$ is a (local) $(P, \mathbb{F})$-martingale. Now, (ii) and (iii) follow from (a) (i) and (ii) using also the fact that $\left(Y_{T}^{2, Q}\right)^{2}$ is $Q$-integrable if and only if it is $Q^{\gamma}$-integrable; this follows from (A.1) for $s=0$ and $X^{2, Q}=\left(Y_{T}^{2, Q}\right)^{2}$ using the fact that a martingale on a finite time horizon is square-integrable if and only if it is square-integrable at the final time.

\section{B Analytic results}

The main objective of this section is to show the existence and uniqueness of a solution to the integral equation (4.13). We first need several preparatory results.

An existence result for ODEs. Let $y \in C[0, T)$ and $U:=\{(t, y) \in[0, T) \times \mathbb{R}: y>y(t)\}$. Let $f: U \rightarrow \mathbb{R}$ be a continuous function that is locally Lipschitz in its second variable. We consider the ordinary differential equation (ODE)

$$
y^{\prime}(t)=f(t, y(t)), \quad t \in[0, T) .
$$

A function $y \in C^{1}[0, T)$ with $y>\underline{y}$ is called a backward upper (lower) solution to (B.1) if

$$
y^{\prime}(t) \leq(\geq) f(t, y(t)), \quad t \in[0, T) .
$$

The function $y$ is called a solution to (B.1) if it is both a backward upper and a backward lower solution.

Remark B.1. We define backward upper and lower solution without an initial condition. Moreover, note that what we call backward upper and lower solutions is called upper and lower solution to the left in Walter (1998). Moreover, in Walter (1998) strict (as opposed to weak) inequalities are considered. But as we require $f$ to be locally Lipschitz continuous in its second variable, all results hold also for the weak inequalities (see Walter, 1998, Corollary VIII.9).

The following result gives the existence of a solution to the ODE (B.1) via the existence of a backward lower and a backward upper solution. The proof for $U=[0, \infty) \times \mathbb{R}$ can be found in Walter (1998, Theorem and Remark XIII.9), and it is straightforward to check that the argument carries over to our setting.

This article is protected by copyright. All rights reserved. 
Lemma B.2. Let $y_{*}, y^{*} \in C^{1}[0, T)$ with $y_{*} \leq y^{*}$. Suppose that $y_{*}$ is a backward lower and $y^{*}$ a backward upper solution to (B.1). Then there exists a solution $y \in C^{1}[0, T)$ to (B.1) with $y_{*} \leq y \leq y^{*}$.

Properties of the auxiliary functions. We collect some analytic properties of the auxiliary functions $a, b, m$, and $n$ defined in (4.7), (4.10), (4.11), and (4.12). If there is no danger of confusion, we drop the dependence on $p$ in the notation. It is easy to check that $a, b, m, n \in$ $C([0, T) \times[-1, \infty) \times(0, \infty)) \cap C^{1,2,1}([0, T) \times(-1, \infty) \times(0, \infty))$. For further reference, we note the straightforward identities

$$
\begin{aligned}
\frac{\partial}{\partial y} a(t, y) & =\frac{1}{p \sigma^{2}} \frac{\phi^{\prime}(t)^{2}}{\kappa^{G}(t)} \geq 0 \\
\frac{\partial}{\partial y} m(t, y) & =(1+y)^{\frac{1}{p}}\left(\frac{1}{p} \frac{a(t, y, p)}{1+y}+\frac{\partial}{\partial y} a(t, y, p)\right) \geq \frac{1}{p} \frac{m(t, y, p)}{1+y}, \\
\frac{\partial}{\partial y} n(t, y) & =\kappa^{G}(t)\left(\frac{1}{p} a(t, y, p)+(1+y) \frac{\partial}{\partial y} a(t, y, p)\right) \geq \frac{1}{p} \kappa^{G}(t) a(t, y, p), \\
n(t, y) & =-\frac{1-p}{2 p^{2} \sigma^{2}} \mu^{2}+\frac{1-p}{2 p^{2} \sigma^{2}}\left(\phi^{\prime}(t) y-\mu\right)^{2}+\frac{1}{p} \kappa^{G}(t)(b(t, y, 1)-1) .
\end{aligned}
$$

In view of the integral equation (4.13), we are interested in the domain where the function $m$ is positive. To this end, define the function $\underline{y}:[0, T) \rightarrow[-1, \infty)$ by

$$
\underline{y}(t):= \begin{cases}-1 & \text { if } \phi^{\prime}(t)=0 \\ \max \left(-1, \frac{\mu}{\phi^{\prime}(t)}-p \sigma^{2} \frac{\kappa^{G}(t)}{\phi^{\prime}(t)^{2}}\right) & \text { if } \phi^{\prime}(t)>0 .\end{cases}
$$

Using that $\kappa^{G}$ is continuous and positive on $[0, T)$, it is not difficult to check that $\underline{y} \in C[0, T)$. Set

$$
U=\{(t, y) \in[0, T) \times \mathbb{R}: y>\underline{y}(t)\} .
$$

Then by the definition of $\underline{y},(B .3)$, and (B.4),

$$
a(t, y), m(t, y), \frac{\partial}{\partial y} m(t, y), \frac{\partial}{\partial y} n(t, y)>0, \quad(t, y) \in U .
$$

An implicit function result. The following inverse-function-type result is the cornerstone of the subsequent analysis. In particular, it is used in Theorem B.5 to construct backward upper and backward lower solutions for the ODE (B.1). Recall the definition of $y$ in (B.6).

Lemma B.3. Fix $p \in(0, \infty)$. Let $f \in C^{1}[0, T)$ with $f(t)>0, t \in[0, T)$. Then there exists a unique function $y \in C^{1}[0, T)$ with $y>\underline{y}$ such that

$$
m(t, y(t))=f(t) .
$$

Moreover, if $\lim _{t \uparrow \uparrow T} f(t)=1$, then there exist constants $\epsilon \in(0,1]$ and $C \geq 1$ such that

$$
\epsilon \leq 1+y(t) \leq C+\frac{C}{\phi^{\prime}(t)} \mathbf{1}_{\left\{\kappa^{G}(t)<C \phi^{\prime}(t)\right\}}, \quad t \in[0, T) .
$$

In this case, if in addition $\int_{0}^{T}\left|\phi^{\prime}(u) y(u)\right| \mathrm{d} u<\infty$, then

$$
\int_{0}^{T} \mathbf{1}_{\{\Delta G(T)>0\}} \kappa^{G}(u)(1+y(u)) \mathrm{d} u<\infty .
$$

This article is protected by copyright. All rights reserved. 
Proof. First, for fixed $t \in[0, T)$, by (4.11) and (B.3), $y \mapsto m(t, y)$ is continuous and increasing ${ }^{17}$ on $[y(t), \infty)$ with $m(t, y(t))=0$ and $\lim _{y \rightarrow \infty} m(t, y)=+\infty$. Thus, there exists a unique function $y:[0, T) \rightarrow \mathbb{R}$ with $y>\underline{y}$ satisfying (B.9). Moreover, $y \in C^{1}(0, T)$ by the implicit function theorem.

Second, for fixed $t \in[0, T)$, we claim that

$$
\begin{array}{ll}
y(t) \leq(2 f(t))^{p} & \text { if } \phi^{\prime}(t) \leq \frac{p \sigma^{2}}{2 \mu} \kappa^{G}(t), \\
y(t) \leq \max \left(f(t)^{p}, \frac{\mu}{\phi^{\prime}(t)}\right) & \text { if } \phi^{\prime}(t)>0 .
\end{array}
$$

Indeed, fix $t \in[0, T)$. If $\phi^{\prime}(t) \leq \frac{p \sigma^{2}}{2 \mu} \kappa^{G}(t)$, then $a(t, 0)=1-\frac{\mu}{p \sigma^{2}} \frac{\phi^{\prime}(t)}{\kappa^{G}(t)} \geq \frac{1}{2}$. Seeking a contradiction, suppose that $y(t)>(2 f(t))^{p}>0$. Then by the definitions of $m$ and $y(t)$ and the monotonicity of $a$ in the second variable,

$$
f(t)=m(t, y(t))=(1+y(t))^{\frac{1}{p}} a(t, y(t))>(y(t))^{\frac{1}{p}} a(t, 0) \geq f(t)
$$

which is absurd. If $\phi^{\prime}(t)>0$, then $a\left(t, \frac{\mu}{\phi^{\prime}(t)}\right)=1$, and (B.13) follows from a similar argument.

Third, by the implicit function theorem and (B.3), for $t \in(0, T)$,

$$
\begin{aligned}
\left|y^{\prime}(t)\right| & =\left|\frac{f^{\prime}(t)-\frac{\partial}{\partial t} m(t, y(t))}{\frac{\partial}{\partial y} m(t, y(t))}\right| \leq p(1+y(t)) \frac{\left|f^{\prime}(t)-\frac{\partial}{\partial t} m(t, y(t))\right|}{m(t, y(t))} \\
& =p(1+y(t)) \frac{\left|f^{\prime}(t)-\frac{\partial}{\partial t} m(t, y(t))\right|}{f(t)} .
\end{aligned}
$$

Now, fix $t_{0} \in(0, T)$ and let $C>0$ be such that $y(t) \leq C$ for all $t \in\left[0, t_{0}\right]$. (This is possible by (B.12), (B.13) and the facts that $f$ is continuous and $\kappa^{G}$ is continuous and positive). Then the positivity and continuity of $f$ in $\left[0, t_{0}\right]$, the continuity of $f^{\prime}$ in $\left[0, t_{0}\right]$, and the continuity of $\frac{\partial}{\partial t} m(t, y)$ in $\left[0, t_{0}\right] \times[-1, C]$ together with the fact that $-1 \leq y(t) \leq C$ for $t \in\left[0, t_{0}\right]$ show that $y^{\prime}$ is uniformly bounded in $\left(0, t_{0}\right]$. Moreover, by the fundamental theorem of calculus and the fact that $y \in C^{1}(0, T)$,

$$
y(t)=y\left(t_{0}\right)-\int_{t}^{t_{0}} y^{\prime}(u) \mathrm{d} u, \quad t \in\left(0, t_{0}\right] .
$$

Thus, by dominated convergence, $\lim _{t \downarrow \downarrow 0} y(t)$ exists in $\mathbb{R}$. The continuity of $f$ and $m$ and (B.9) give

$$
m(0, y(0))=f(0)=\lim _{t \downarrow \downarrow 0} f(t)=\lim _{t \downarrow \downarrow 0} m(t, y(t))=m\left(0, \lim _{t \downarrow \downarrow 0} y(t)\right),
$$

and so by the uniqueness of $y$ on $[0, T), \lim _{t \downarrow \downarrow 0} y(t)=y(0)>y(0) \geq-1$. This together with the continuity of $\frac{\partial}{\partial y} m$ and $\frac{\partial}{\partial t} m$ on $[0, T) \times(-1, \infty)$, the continuity of $f^{\prime}$ on $[0, T)$, and the identity $y^{\prime}(t)=\left(f^{\prime}(t)-\frac{\partial}{\partial t} m(t, y(t))\right) / \frac{\partial}{\partial y} m(t, y(t))$ for $t \in(0, T)$ (by the implicit function theorem) shows that the $\operatorname{limit}_{t \downarrow \lim _{t \downarrow 0}} y^{\prime}(t)$ exists in $\mathbb{R}$. So $y \in C^{1}[0, T)$.

Fourth, assume $\lim _{t \uparrow \uparrow T} f(t)=1$. Set

$$
C:=1+\max \left(\sup _{t \in[0, T)}(2 f(t))^{p}, \mu, \frac{2 \mu}{p \sigma^{2}}\right) .
$$

Then $1 \leq C<\infty$. Fix $t \in[0, T)$. If $\kappa^{G}(t) \geq C \phi^{\prime}(t)$, then $\phi^{\prime}(t) \leq \frac{1}{C} \kappa^{G}(t) \leq \frac{p \sigma^{2}}{2 \mu} \kappa^{G}(t)$, and so $1+y(t) \leq C$ by (B.12) and the definition of $C$. Otherwise, if $\kappa^{G}(t)<C \phi^{\prime}(t)$, then $\phi^{\prime}(t)>0$,

\footnotetext{
${ }^{17}$ We emphasize that we use qualifiers like "increasing", "decreasing", "positive", "negative" in the strict sense; the corresponding wide-sense notions are "nondecreasing", "nonincreasing", "nonnegative", "nonpositive".
}

This article is protected by copyright. All rights reserved. 
and so $1+y(t) \leq C+\frac{C}{\phi^{\prime}(t)}$ by (B.13) and the definition of $C$. For the left inequality in (B.10), by the continuity of $y$ in $[0, T)$ and the fact that $y>y \geq-1$ on $[0, T)$, it suffices to show that $\liminf \operatorname{i\uparrow \uparrow T}_{t \uparrow} y(t)>-1$. Seeking a contradiction, suppose there is a sequence $\left(t_{n}\right)_{n \in \mathbb{N}} \subset[0, T)$ increasing to $T$ such that $\lim _{n \rightarrow \infty} y\left(t_{n}\right)=-1$. Passing to a subsequence if necessary, we may assume that $y\left(t_{n}\right) \leq 0$ for all $n \in \mathbb{N}$. As $\phi^{\prime} \geq 0$ by (2.5), the definition of $a$ in (4.7) gives $a\left(t_{n}, y\left(t_{n}\right)\right) \leq 1$ for all $n \in \mathbb{N}$. Now, using the definition of $m$ in (4.11), we arrive at the contradiction

$$
1=\lim _{n \rightarrow \infty} f\left(t_{n}\right)=\lim _{n \rightarrow \infty} m\left(t_{n}, y\left(t_{n}\right)\right) \leq \limsup _{n \rightarrow \infty}\left(1+y\left(t_{n}\right)\right)^{\frac{1}{p}}=0 .
$$

Finally, assume that in addition $\Delta G(T)>0$ and $\int_{0}^{T}\left|\phi^{\prime}(u) y(u)\right| \mathrm{d} u<\infty$. Then by (2.1),

$$
\int_{0}^{T} \kappa^{G}(u) \mathrm{d} u=-\log (\Delta G(T))<\infty .
$$

Define $C$ as in (B.14), and set $A:=\left\{u \in[0, T): \phi^{\prime}(u) \leq \frac{p \sigma^{2}}{2 \mu} \kappa^{G}(u)\right\}$. Then $y(u) \leq C$ for $u \in A$ by (B.12), and $\kappa^{G}(u)<\frac{2 \mu}{p \sigma^{2}} \phi^{\prime}(u)$ for $u \in A^{c}$. This together with the above yields (B.11) via

$$
\begin{aligned}
\int_{0}^{T}(1+y(u)) \kappa^{G}(u) \mathrm{d} u & =\int_{0}^{T}\left(\kappa^{G}(u)+\mathbf{1}_{A}(u) y(u) \kappa^{G}(u)+\mathbf{1}_{A^{c}}(u) y(u) \kappa^{G}(u)\right) \mathrm{d} u \\
& \leq \int_{0}^{T}\left((1+C) \kappa^{G}(u)+\frac{2 \mu}{p \sigma^{2}} \phi^{\prime}(u)|y(u)|\right) \mathrm{d} u<\infty .
\end{aligned}
$$

Corollary B.4. Fix $p \in(0, \infty)$. Let $f, g \in C^{1}[0, T)$ be such that $g(t)>f(t)>0, t \in[0, T)$, and $\lim _{t \uparrow \uparrow T} g(t)=\lim _{t \uparrow \uparrow T} f(t)=1$. Let $y^{f}, y^{g} \in C^{1}[0, T)$ with $y^{g}, y^{f}>y$ be the unique functions from Lemma B.3 satisfying $m\left(t, y^{f}(t)\right)=f(t)$ and $m\left(t, y^{g}(t)\right)=g(t)$. Assume that $\Delta G(T)>0$ and $\lim \sup _{t \uparrow \uparrow T} G^{\prime}(t)<\infty$. Then

$$
\lim _{t \uparrow \uparrow T} \phi^{\prime}(t)\left(y^{g}(t)-y^{f}(t)\right)=0
$$

Proof. By Lemma B.3, there are constants $C^{g}, \epsilon^{f}>0$ such that $1+y^{g}(t) \leq C^{g}+\frac{C^{g}}{\phi^{\prime}(t)} \mathbf{1}_{\left\{\phi^{\prime}(t)>0\right\}}$ and $1+y^{f}(t) \geq \epsilon^{f}, t \in[0, T)$. As $\Delta G(T)>0$ and $\limsup _{t \uparrow \uparrow T} G^{\prime}(t)<\infty$, there exists a constant $C^{\kappa}>0$ such that $\frac{1}{\kappa^{G}(t)}=\frac{1-G(t)}{G^{\prime}(t)} \geq C^{\kappa}, t \in[0, T)$. Next, as $f \in C^{1}[0, T), f>0$ on $[0, T)$, and $\lim _{t \uparrow \uparrow T} f(t)=1$, there exists a constant $C^{f}>0$ such that $f(t) \geq C^{f}, t \in[0, T)$. Finally, set $C=\min \left(\frac{C^{f}}{2 C^{g}}, \frac{\left(\epsilon^{f}\right)^{\frac{1}{p}} C^{\kappa}}{\sigma^{2}}\right)>0$. Fix $t \in[0, T)$. Then $y^{g}(t) \geq y^{f}(t)$ by the monotonicity of $m$ in the second variable. Using successively (B.9), the mean value theorem, (B.3), (4.11) and (B.2), the monotonicity of $m$ in the second variable, (B.9) and the choices of $C^{g}, \epsilon^{f}$, and $C^{\kappa}$, the choice of $C^{f}$, and finally the choice of $C$ (distinguishing the cases $\phi^{\prime}(t) \geq 1$ and $\phi^{\prime}(t)<1$ ) yields, for some

This article is protected by copyright. All rights reserved. 
$\tilde{y} \in\left[y^{f}(t), y^{g}(t)\right]$,

$$
\begin{aligned}
g(t)-f(t) & =m\left(t, y^{g}(t)\right)-m\left(t, y^{f}(t)\right)=\left(y^{g}(t)-y^{f}(t)\right) \frac{\partial}{\partial y} m(t, \tilde{y}) \\
& =\left(y^{g}(t)-y^{f}(t)\right)(1+\tilde{y})^{\frac{1}{p}}\left(\frac{1}{p} \frac{a(t, \tilde{y})}{1+\tilde{y}}+\frac{\partial}{\partial y} a(t, \tilde{y})\right) \\
& =\frac{1}{p}\left(y^{g}(t)-y^{f}(t)\right)\left(\frac{m(t, \tilde{y})}{1+\tilde{y}}+\frac{1}{\sigma^{2}}(1+\tilde{y})^{\frac{1}{p}} \frac{\phi^{\prime}(t)^{2}}{\kappa^{G}(t)}\right) \\
& \geq \frac{1}{p}\left(y^{g}(t)-y^{f}(t)\right)\left(\frac{m\left(t, y^{f}(t)\right)}{1+y^{g}(t)}+\frac{1}{\sigma^{2}}\left(1+y^{f}(t)\right)^{\frac{1}{p}} \frac{\phi^{\prime}(t)^{2}}{\kappa^{G}(t)}\right) \\
& \geq \frac{1}{p}\left(y^{g}(t)-y^{f}(t)\right)\left(\frac{f(t)}{C^{g}+\frac{C^{g}}{\phi^{\prime}(t)} \mathbf{1}_{\left\{\phi^{\prime}(t)>0\right\}}}+\frac{\left(\epsilon^{f}\right)^{\frac{1}{p}} C^{\kappa}}{\sigma^{2}} \phi^{\prime}(t)^{2}\right) \\
& \geq \frac{1}{p} \phi^{\prime}(t)\left(y^{g}(t)-y^{f}(t)\right)\left(\frac{C^{f}}{C^{g} \phi^{\prime}(t)+C^{g}}+\frac{\left(\epsilon^{f}\right)^{\frac{1}{p}} C^{\kappa}}{\sigma^{2}} \phi^{\prime}(t)\right) \\
& \geq \frac{C}{p} \phi^{\prime}(t)\left(y^{g}(t)-y^{f}(t)\right) .
\end{aligned}
$$

Now, the claim follows from letting $t \uparrow \uparrow T$.

Existence and uniqueness of a solution to the integral equation. We are now in a position to prove the main existence and uniqueness result for the integral equation (4.13). Recall the definition of $\underline{y}$ in (B.6).

Theorem B.5. Fix $p \in(0, \infty)$. Then there exists a unique solution $\hat{y} \in C^{1}[0, T)$ with $\hat{y}>\underline{y}$ to the integral equation

$$
m(t, y(t), p)=\exp \left(-\int_{t}^{T} n(u, y(u), p) \mathrm{d} u\right), \quad t \in[0, T),
$$

satisfying (B.10) and (B.11) (with y replaced by $\hat{y}$ ) as well as

$$
\int_{0}^{T}|n(u, \hat{y}(u), p)| \mathrm{d} u<\infty \quad \text { and } \quad \int_{0}^{T}\left(\phi^{\prime}(u) \hat{y}(u)\right)^{2} \mathrm{~d} u<\infty .
$$

Moreover, $y_{*} \leq \hat{y} \leq y^{*}$ on $[0, T)$, where $y_{*}, y^{*} \in C^{1}[0, T)$ are the unique functions from Lemma B.3 satisfying $y_{*}, y^{*}>\underline{y}$ and

$$
\begin{aligned}
& m\left(t, y^{*}(t), p\right)= \begin{cases}\exp \left(\frac{1-p}{2 p^{2} \sigma^{2}} \mu^{2}(T-t)\right) & \text { if } p<1, \\
1 & \text { if } p \geq 1,\end{cases} \\
& m\left(t, y_{*}(t), p\right)= \begin{cases}1 & \text { if } p<1, \\
\exp \left(\frac{1-p}{2 p^{2} \sigma^{2}} \mu^{2}(T-t)\right) & \text { if } p \geq 1\end{cases}
\end{aligned}
$$

Note that (B.10) and (B.11) (with $y$ replaced by $\hat{y}$ ) as well as (B.16) imply in particular that (3.12) and (3.18) (with $y$ replaced by $\hat{y}$ ) are fulfilled.

Proof. First, we transform the integral equation (B.15) into an ODE. Taking logarithms on both sides of (B.15) and differentiating shows that a solution $y \in C^{1}[0, T)$ to (4.13) solves

$$
\frac{\mathrm{d}}{\mathrm{d} t} \log (m(t, y(t)), p)=n(t, y(t), p) .
$$

This article is protected by copyright. All rights reserved. 
An easy calculation using (4.11) and (B.3) gives

$$
\frac{\mathrm{d}}{\mathrm{d} t} \log (m(t, y(t), p))=\frac{y^{\prime}(t)\left(\frac{1}{p} \frac{a(t, y(t), p)}{1+y(t)}+\frac{\partial}{\partial y} a(t, y(t), p)\right)+\frac{\partial}{\partial t} a(t, y(t), p)}{a(t, y(t), p)} .
$$

Rearranging the terms shows that $y$ solves the ODE

$$
y^{\prime}(t)=f(t, y(t), p), \quad t \in[0, T),
$$

where the function $f: U \times(0, \infty) \rightarrow(0, \infty)$ is given by

$$
f(t, y, p)=\frac{a(t, y, p) n(t, y, p)-\frac{\partial}{\partial t} a(t, y, p)}{\frac{1}{p} \frac{a(t, y, p)}{1+y}+\frac{\partial}{\partial y} a(t, y, p)}
$$

and $U$ is defined in (B.7). Clearly, $f \in C^{0,1,1}(U \times(0, \infty))$. Note that the positivity of the denominator is ensured by the positivity of $a$ in $U \times(0, \infty)$ by (B.8) and (B.2). Moreover, (B.15) gives the implicit "terminal condition"

$$
\lim _{t \uparrow \uparrow T} m(t, y(t), p)=1 .
$$

Second, we establish the uniqueness of $\hat{y}$. Assume that $\hat{y}^{1}, \hat{y}^{2} \in C^{1}[0, T)$ are two solutions of (B.15). Then $\hat{y}^{1}, \hat{y}^{2}>y$ and both functions are solutions to the ODE (B.20). Assume without loss of generality that $\overline{\hat{y}}^{2}(0) \geq \hat{y}^{1}(0)$. As $f$ is locally Lipschitz in the second variable on $U$, it follows from the standard local existence and uniqueness theorem for ODEs that either $\hat{y}^{1}=\hat{y}^{2}$ or $\hat{y}^{2}>\hat{y}^{1}$. Seeking a contradiction, assume the second case. Then by the strict monotonicity of $m$ and $n$ in the second variable (by (B.8)) and the fact that $\hat{y}^{1}$ and $\hat{y}^{2}$ are solutions to (4.13),

$$
\begin{aligned}
m\left(0, \hat{y}^{2}(0)\right) & >m\left(0, \hat{y}^{1}(0)\right)=\exp \left(-\int_{0}^{T} n\left(u, \hat{y}^{1}(u)\right) \mathrm{d} u\right) \\
& >\exp \left(-\int_{0}^{T} n\left(u, \hat{y}^{2}(u)\right) \mathrm{d} u\right)=m\left(0, \hat{y}^{2}(0)\right),
\end{aligned}
$$

which is absurd. So $\hat{y}^{1}=\hat{y}^{2}$.

Third, we use Lemma B.2 to show the existence of a solution to (B.20). To this end, we show that $y^{*}$ and $y_{*}$ are backward upper and backward lower solutions, respectively. The existence and uniqueness of the functions $y_{*}$ and $y^{*}$ satisfying (B.17) and (B.18) follows from Lemma B.3. Note that $y \in C^{1}[0, T)$ with $y>\underline{y}$ is a backward upper (lower) solution to (B.20) if and only if

$$
\frac{\mathrm{d}}{\mathrm{d} t} \log (m(t, y(t), p)) \leq(\geq) n(t, y(t), p), \quad t \in[0, T) ;
$$

this follows from the same rearrangement that led from (B.19) to (B.20) using that $\frac{1}{p} \frac{a}{1+y}+\frac{\partial}{\partial y} a$ and $a$ are positive in $U \times(0, \infty)$ by (B.8) and (B.2).

We only consider the case $p<1$; the case $p \geq 1$ follows from a similar argument, basically reversing all inequalities. Bernoulli's inequality, (4.10), and (4.11) yield

$$
b(t, y, p) \leq m(t, y, p) \leq b(t, y, 1)^{1 / p}, \quad(t, y) \in U .
$$

To establish that $y^{*}$ is a backward upper solution, note from (B.17) that $m\left(t, y^{*}(t), p\right) \geq 1$ for $t \in[0, T)$. Thus, $b\left(t, y^{*}(t), 1\right) \geq 1$ for $t \in[0, T)$ by (B.22), and so $n\left(t, y^{*}(t), p\right) \geq-\frac{1-p}{2 p^{2} \sigma^{2}} \mu^{2}$ for $t \in[0, T)$ by (B.5). Now, taking logarithms in (B.17) and differentiating shows that $y^{*}$ fulfills (B.21) with " $\leq$ ", and so $y^{*}$ is a backward upper solution. To establish that $y_{*}$ is a backward lower solution, note from (B.18) that $m\left(t, y_{*}(t), p\right)=1$ for $t \in[0, T)$, and so $b\left(t, y_{*}(t), p\right) \leq 1$ for $t \in[0, T)$ by (B.22). Thus, $n\left(t, y_{*}(t), p\right) \leq 0$ by (4.12), and the claim follows as above by taking logarithms

This article is protected by copyright. All rights reserved. 
in (B.18) and differentiating. Clearly, $y_{*} \leq y^{*}$ by the monotonicity of $m$ in in the second variable, and $\lim _{t \uparrow \uparrow T} m\left(t, y_{*}(t), p\right)=\lim _{t \uparrow \uparrow T} m\left(t, y^{*}(t), p\right)=1$ by construction. So by Lemma B.2, there exists a solution $\hat{y} \in C^{1}[0, T)$ of (B.20) with $y_{*} \leq \hat{y} \leq y^{*}$.

Fourth, $\hat{y}>\underline{y}$ because $\hat{y} \geq y_{*}>y$ by construction. The monotonicity of $m$ in the second variable and the fact that $\lim _{t \uparrow \uparrow T} m\left(t, \bar{y}^{*}(t), p\right)=1=\lim _{t \uparrow \uparrow T} m\left(t, y_{*}(t), p\right)$ by (B.17) and (B.18) yield $\lim _{t \uparrow \uparrow T} m(t, \hat{y}(t), p)=1$. Moreover, as $\hat{y}$ satisfies (B.19), the fundamental theorem of calculus shows that there exists a constant $c>0$ such that $\hat{y}$ satisfies the integral equation

$$
m(t, \hat{y}(t), p)=c \exp \left(\int_{0}^{t} n(u, \hat{y}(u), p) \mathrm{d} u\right), \quad t \in[0, T) .
$$

Now, we have to distinguish the cases $p<1$ and $p \geq 1$. We only consider the first one; the second one follows from a similar argument, basically reversing all inequalities. So let $p \in(0,1)$. Then $m(t, \hat{y}(t), p) \geq m\left(t, y_{*}(t), p\right)=1$ by the monotonicity of $m$ in the second variable and (B.18). Thus, (B.22) gives $b(t, \hat{y}(t), 1) \geq 1$, and so $n(t, \hat{y}(t), p) \geq-\frac{1-p}{2 p^{2} \sigma^{2}} \mu^{2}$ by (B.5). Taking the limit $t \uparrow \uparrow T$ in (B.23), by monotone convergence and the fact that $\lim _{t \uparrow \uparrow T} m(t, \hat{y}(t), p)=1$, we deduce that

$$
c=\exp \left(-\int_{0}^{T} n(u, \hat{y}(u), p) \mathrm{d} u\right) .
$$

Plugging this back into (B.23) shows that $\hat{y}$ is a solution to (B.15). Moreover, as $n(t, \hat{y}(t), p)$ is bounded from below and $c>0,($ B.24) implies that the first condition in (B.16) is satisfied. This together with the representation of $n$ in (B.5) and $b(t, \hat{y}(t), 1) \geq 1$ (from above) then also establishes the second condition in (B.16). Finally, define $\hat{f}(t)$ by the right-hand side of (B.15) (with $y$ replaced by $\hat{y})$. Then $\hat{y}$ is trivially a solution to $m(t, \hat{y}(t))=\hat{f}(t), t \in[0, T)$, and $\lim _{t \uparrow \uparrow T} \hat{f}(t)=1$. Hence, Lemma B.3 gives (B.10) and (B.11) for $\hat{y}$ (note that the condition $\int_{0}^{T}\left|\phi^{\prime}(u) \hat{y}(u)\right| \mathrm{d} u<\infty$ follows from (B.16)).

\section{Verification}

Here, we collect the technical parts of Steps 2 and 3 of the proof of Theorem 4.2. The first result identifies the wealth process corresponding to the strategy $\hat{\pi}$ and shows that it remains positive. The second and third result verify $(\mathrm{OC} 1)$ and $(\mathrm{OC} 2)$ for the candidate triplet $(\hat{\pi}, \hat{Q}, \hat{z})$.

Lemma C.1. Let $(\hat{\pi}, \hat{Q}, \hat{z})$ be the triplet defined in (the proof of) Theorem 4.2. Denote by $W^{\hat{Q}}$ the $\hat{Q}$-Brownian motion given by Theorem 3.4 (with $y=\hat{y}$ ) and let $\hat{H}$ be the distribution function of $\gamma$ under $\hat{Q}$. Define $\hat{\xi} \in C^{1}[0, T)$ by

$$
\hat{\xi}(t)=\exp \left(\int_{0}^{t} \phi^{\prime}(u) \frac{1}{p \sigma^{2}}\left(\mu-\phi^{\prime}(u) \hat{y}(u)\right)(1+\hat{y}(u)) \mathrm{d} u\right)
$$

and set $\hat{X}:=\mathcal{E}\left(\sigma \int_{0}^{\cdot} \hat{\pi}_{t} \mathrm{~d} W_{t}^{\hat{Q}}\right) \mathcal{M}^{\hat{H}} \hat{\xi}$. Then $X^{\hat{\pi}}=x \hat{X}$ is the wealth process corresponding to the strategy $\hat{\pi}$ and initial capital $x$. Moreover, $\mathcal{M}^{\hat{H}} \hat{\xi}$ and $\hat{X}$ are positive and thus $\hat{\pi}$ is admissible.

Proof. For the first claim, it suffices to show that $\hat{X}$ satisfies the SDE (4.1) with initial condition $\hat{X}_{0}=1$. Set $M:=\mathcal{E}\left(\sigma \int_{0}^{\cdot} \hat{\pi}_{t} \mathrm{~d} W_{t}^{\hat{Q}}\right)$ and $N:=\mathcal{M}^{\hat{H}} \hat{\xi}$ for brevity and note from (4.14) that $\hat{\pi}_{t}=\bar{\pi}(t, \gamma), t \in[0, T]$, where

$$
\bar{\pi}(t, v):=\frac{1}{p \sigma^{2}}\left(\mu-\phi^{\prime}(t) \hat{y}(t) \mathbf{1}_{\{t \leq v, t<T\}}\right), \quad(t, v) \in[0, T]^{2} .
$$

With this notation, by the definition of $\hat{\xi}$, we obtain

$$
\hat{\xi}^{\prime}(t)=\hat{\xi}(t) \phi^{\prime}(t) \bar{\pi}(t, t)(1+\hat{y}(t)), \quad t \in[0, T) .
$$

This article is protected by copyright. All rights reserved. 
Fix $t \in[0, T]$. By using successively that $M$ is continuous and $N$ is purely discontinuous, that $\Delta \mathcal{M}^{\hat{H}} \hat{\xi}_{\gamma}=-\frac{\hat{\xi}^{\prime}(\gamma)}{\kappa^{\hat{H}}(\gamma)} \mathbf{1}_{\{\gamma<T\}}$ by the definitions of $\mathcal{M}^{\hat{H}} \hat{\xi}$ (cf. (2.2)) and $\mathcal{A}^{\hat{H}} \hat{\xi}$ (cf. (2.3)), (C.3), that $\hat{\xi}(s)=N_{s-}$ and $\bar{\pi}(s, s)=\bar{\pi}(s, \gamma)=\hat{\pi}_{s}$ on $\{s \leq \gamma\}$, and finally the dynamics of $S$ in (3.16) (for $y=\hat{y}$ etc.),

$$
\begin{aligned}
& \hat{X}_{t}-1=M_{t} N_{t}-M_{0} N_{0}=\int_{0}^{t} N_{s-} \mathrm{d} M_{s}+\int_{0}^{t} M_{s-} \mathrm{d} N_{s} \\
& =\sigma \int_{0}^{t} N_{s-} M_{s-} \hat{\pi}_{s} \mathrm{~d} W_{s}^{\hat{Q}}+\int_{0}^{t} M_{s-} \mathrm{d} \mathcal{M}^{\hat{H}} \hat{\xi}_{s} \\
& =\sigma \int_{0}^{t} \hat{X}_{s-} \hat{\pi}_{s} \mathrm{~d} W_{s}^{\hat{Q}}+\int_{0}^{t \wedge \gamma} M_{s-} \hat{\xi}^{\prime}(s) \mathrm{d} s+M_{\gamma-} \Delta \mathcal{M}^{\hat{H}} \hat{\xi}_{\gamma} \mathbf{1}_{\{\gamma \leq t\}} \\
& =\sigma \int_{0}^{t} \hat{X}_{s-} \hat{\pi}_{s} \mathrm{~d} W_{s}^{\hat{Q}}+\int_{0}^{t \wedge \gamma} M_{s-} \hat{\xi}^{\prime}(s) \mathrm{d} s-M_{\gamma-} \frac{\hat{\xi}^{\prime}(\gamma)}{\kappa^{\hat{H}}(\gamma)} \mathbf{1}_{\{\gamma \leq t, \gamma<T\}} \\
& =\sigma \int_{0}^{t} \hat{X}_{s-} \hat{\pi}_{s} \mathrm{~d} W_{s}^{\hat{Q}}+\int_{0}^{t \wedge \gamma} M_{s-} \hat{\xi}(s) \bar{\pi}(s, s) \phi^{\prime}(s)(1+\hat{y}(s)) \mathrm{d} s
\end{aligned}
$$

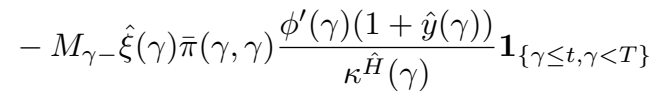

$$
\begin{aligned}
& =\sigma \int_{0}^{t} \hat{X}_{s-} \hat{\pi}_{s} \mathrm{~d} W_{s}^{\hat{Q}}+\int_{0}^{t \wedge \gamma} M_{s-} N_{s-} \hat{\pi}_{s} \phi^{\prime}(s)(1+\hat{y}(s)) \mathrm{d} s \\
& +M_{\gamma-} N_{\gamma-} \hat{\pi}_{\gamma} \Delta \mathcal{M}^{\hat{H}}\left(\int_{0}^{\cdot} \phi^{\prime}(u)(1+\hat{y}(u)) \mathrm{d} u\right)_{\gamma} \mathbf{1}_{\{\gamma \leq t\}} \\
& =\int_{0}^{t} \hat{\pi}_{s} \hat{X}_{s-}\left[\sigma \mathrm{d} W_{s}^{\hat{Q}}+\mathrm{d} \mathcal{M}^{\hat{H}}\left(\int_{0}^{\cdot} \phi^{\prime}(u)(1+\hat{y}(u)) \mathrm{d} u\right)_{s}\right] \\
& =\int_{0}^{t} \hat{\pi}_{s} \hat{X}_{s-} \frac{\mathrm{d} S_{s}}{S_{s-}} \quad P \text {-a.s. }
\end{aligned}
$$

For the second claim, as $M$ and $\hat{\xi}$ are positive, it suffices to show that also $\mathcal{A}^{\hat{H}} \hat{\xi}$ is positive. Indeed, using the definition of $\mathcal{A}^{\hat{H}} \hat{\xi}\left(\operatorname{cf}\right.$. (2.3)), (C.3), (3.6), the fact that $a(v, \hat{y}(v), p)=1-\frac{\phi^{\prime}(v)}{\kappa^{G}(v)} \bar{\pi}(v, v)$ by the definitions of $\bar{\pi}$ and $a$ in (C.2) and (4.7), and (B.8),

$$
\mathcal{A}^{\hat{H}} \hat{\xi}(v)=\hat{\xi}(v)-\frac{\hat{\xi}^{\prime}(v)}{\kappa^{\hat{H}}(v)}=\hat{\xi}(v)\left(1-\bar{\pi}(v, v) \frac{\phi^{\prime}(v)}{\kappa^{G}(v)}\right)=\hat{\xi}(v) a(v, \hat{y}(v), p)>0, \quad v \in(0, T) .
$$

Lemma C.2. The triplet $(\hat{\pi}, \hat{Q}, \hat{z})$ defined in the proof of Theorem 4.2 satisfies $U^{\prime}\left(X_{T}^{\hat{\pi}}\right)=\hat{z} \frac{\mathrm{d} \hat{Q}}{\mathrm{~d} P}$.

Proof. By Lemma C.1 and the fact that $U^{\prime}(x)=x^{-p}$,

$$
U^{\prime}\left(X_{T}^{\hat{\pi}}\right)=x^{-p} \hat{X}_{T}^{-p}=x^{-p} \mathcal{E}_{T}\left(\sigma \int_{0}^{\cdot} \hat{\pi}_{t} \mathrm{~d} W_{t}^{\hat{Q}}\right)^{-p}\left(\mathcal{M}_{T}^{\hat{H}} \hat{\xi}\right)^{-p} .
$$

First, a standard calculation gives

$$
\mathcal{E}_{T}\left(\sigma \int_{0}^{\cdot} \hat{\pi}_{t} \mathrm{~d} W_{t}^{\hat{Q}}\right)^{-p}=\mathcal{E}_{T}\left(-p \sigma \int_{0}^{\cdot} \hat{\pi}_{t} \mathrm{~d} W_{t}\right) \exp \left((1-p) \frac{p \sigma^{2}}{2} \int_{0}^{T} \hat{\pi}_{t}^{2} \mathrm{~d} t\right) .
$$

To compute the second factor, we claim that for $v \in[0, T)$,

$$
\hat{\xi}(v) m(v, \hat{y}(v), p)=x^{-1} \hat{z}^{-\frac{1}{p}} \exp \left((1-p) \frac{\sigma^{2}}{2} \int_{0}^{T} \bar{\pi}(u, v)^{2} \mathrm{~d} u\right) \hat{\zeta}(v)^{-\frac{1}{p}}
$$

This article is protected by copyright. All rights reserved. 
where $\bar{\pi}$ is defined in (C.2) and $\hat{\zeta}$ is given in (3.13) (with $y$ replaced by $\hat{y}$ ). Moreover, in the case $\Delta G(T)>0$, we claim that

$$
\hat{\xi}(T-)=x^{-1} \hat{z}^{-\frac{1}{p}} \exp \left((1-p) \frac{\sigma^{2}}{2} \int_{0}^{T} \bar{\pi}(u, u)^{2} \mathrm{~d} u\right) \hat{\zeta}(T-)^{-\frac{1}{p}} .
$$

Then, by (C.4), the definition of $m$ in (4.11), (C.7), and (3.4), on $\{\gamma<T\}$,

$$
\begin{aligned}
\left(\mathcal{M}_{T}^{\hat{H}} \hat{\xi}\right)^{-p} & =\left(\mathcal{A}^{\hat{H}} \hat{\xi}(\gamma)\right)^{-p}=\hat{\xi}(\gamma)^{-p} a(\gamma, \hat{y}(\gamma), p)^{-p} \\
& =(\hat{\xi}(\gamma) m(\gamma, \hat{y}(\gamma), p))^{-p}(1+\hat{y}(\gamma)) \\
& =x^{p} \hat{z} \exp \left((1-p) \frac{\sigma^{2}}{2} \int_{0}^{T} \hat{\pi}_{t}^{2} \mathrm{~d} t\right)^{-p} \hat{\zeta}(\gamma)(1+\hat{y}(\gamma)) \\
& =x^{p} \hat{z} \exp \left(-(1-p) \frac{p \sigma^{2}}{2} \int_{0}^{T} \hat{\pi}_{t}^{2} \mathrm{~d} t\right) \mathcal{A}^{G} \hat{\zeta}(\gamma) .
\end{aligned}
$$

If $\Delta G(T)>0$, then $\mathcal{A}^{\hat{H}} \hat{\xi}(\gamma)=\hat{\xi}(T-)$ and $\mathcal{A}^{G} \hat{\zeta}(\gamma)=\hat{\zeta}(T-)$ on $\{\gamma=T\}$. This together with (C.8) shows that (C.9) holds on $\{\gamma=T\}$, too.

Finally, plugging (C.6) and (C.9) into (C.5) yields by the definitions of $\hat{\pi}$ in (4.14) and $\frac{\mathrm{d} \hat{Q}}{\mathrm{~d} P}$ in Theorem 3.4 (cf. (3.15)) that

$$
\begin{aligned}
U^{\prime}\left(X_{T}^{\hat{\pi}}\right) & =\hat{z} \mathcal{E}_{T}\left(-p \sigma \int_{0}^{\cdot} \hat{\pi}_{t} \mathrm{~d} W_{t}\right) \mathcal{A}^{G} \hat{\zeta}(\gamma)=\hat{z} \mathcal{E}_{T}\left(-p \sigma \int_{0} \hat{\pi}_{t} \mathrm{~d} W_{t}\right) \mathcal{M}_{T}^{G} \hat{\zeta} \\
& =\hat{z} \mathcal{E}_{T}\left(-\int_{0} \frac{1}{\sigma}\left(\mu-\phi^{\prime}(t) \hat{y}(t) \mathbf{1}_{\{t \leq \gamma, t<T\}}\right) \mathrm{d} W_{t}\right) \mathcal{M}_{T}^{G} \hat{\zeta}=\hat{z} \frac{\mathrm{d} \hat{Q}}{\mathrm{~d} P} .
\end{aligned}
$$

It remains to show (C.7) and (C.8). First, an easy but tedious calculation using the definitions of $\hat{\pi}$ and $n$ in (C.2) and (4.12) shows that for $u \in[0, T)$,

$$
\phi^{\prime}(u) \bar{\pi}(u, u)(1+\hat{y}(u))+n(u, \hat{y}(u), p)=\frac{1-p}{2 p^{2} \sigma^{2}} \phi^{\prime}(u) \hat{y}(u)\left(\phi^{\prime}(u) \hat{y}(u)-2 \mu\right)+\frac{1}{p} \kappa^{G}(u) \hat{y}(u) .
$$

Next, fix $v \in[0, T)$. Using first that $\hat{y}$ is a solution to the integral equation (4.13) and the definitions of $\hat{\xi}$ in (C.1) and $\bar{\pi}$ in (C.2), then (C.10), and finally again the definition of $\bar{\pi}$ and the definition of $\hat{\zeta}$ in $(3.13)$ (with $y$ replaced by $\hat{y}$ ),

$$
\begin{aligned}
\hat{\xi}(v) \frac{m(v, \hat{y}(v), p)}{m(0, \hat{y}(0), p)} & =\exp \left(\int_{0}^{v}\left(\phi^{\prime}(u) \bar{\pi}(u, u)(1+\hat{y}(u))+n(u, \hat{y}(u), p)\right) \mathrm{d} u\right) \\
& =\exp \left(\int_{0}^{v}\left(\frac{1-p}{2 p^{2} \sigma^{2}} \phi^{\prime}(u) \hat{y}(u)\left(\phi^{\prime}(u) \hat{y}(u)-2 \mu\right)+\frac{1}{p} \kappa^{G}(u) \hat{y}(u)\right) \mathrm{d} u\right) \\
& =\exp \left(-\frac{1-p}{2 p^{2} \sigma^{2}} \mu^{2} T+(1-p) \frac{\sigma^{2}}{2} \int_{0}^{T} \bar{\pi}(u, v)^{2} \mathrm{~d} u\right) \hat{\zeta}(v)^{-\frac{1}{p}},
\end{aligned}
$$

and using the definition of $\hat{z}$ in (4.17) gives (C.7).

Finally, assume that $\Delta G(T)>0$. Then also $\Delta \hat{H}(T)>0$ as $\hat{Q} \approx P$. Moreover, by the proof of Theorem 3.4, $\mathcal{M}^{G} \hat{\zeta}$ is positive, and by Lemma C.1, $\mathcal{M}^{\hat{H}} \hat{\xi}$ is positive. This together with Proposition 2.2 (a) and (b) (i) implies that the limits $\hat{\zeta}(T-)$ and $\hat{\xi}(T-)$ exist in $\mathbb{R}$. Moreover, $\lim _{v \uparrow \uparrow T} m(v, \hat{y}(v), p)=1$ by (4.13) (recall that Theorem B.5 shows that $\hat{y}$ is a solution to the integral equation) and thus (C.8) follows from taking the limit $v \uparrow \uparrow T$ in (C.7); the exchange of limit and integration on the right-hand side is justified by dominated convergence using the estimate $|\bar{\pi}(u, v)| \leq \frac{1}{p \sigma^{2}}\left(\mu+\left|\phi^{\prime}(u) \hat{y}(u)\right|\right)$ and (B.16); also note that for $u \in[0, T), \lim _{v \uparrow \uparrow T} \bar{\pi}(u, v)=$ $\frac{1}{p \sigma^{2}}\left(\mu-\phi^{\prime}(u) \hat{y}(u)\right)=\bar{\pi}(u, u)$.

This article is protected by copyright. All rights reserved. 
Lemma C.3. The triplet $(\hat{\pi}, \hat{Q}, \hat{z})$ defined in the proof of Theorem 4.2 satisfies $E^{\hat{Q}}\left[X_{T}^{\hat{\pi}}\right]=x$.

Proof. It suffices to show that $X^{\hat{\pi}}$ is a $\hat{Q}$-martingale. Lemma C. 1 shows that $X^{\hat{\pi}}$ is of the form (3.17). Therefore, by Corollary 3.5, it suffices to prove that $\int_{0}^{T}\left|\mathcal{A}^{\hat{H}} \hat{\xi}(u)\right| \hat{H}^{\prime}(u) \mathrm{d} u<\infty$ and that $\mathcal{M}^{\hat{H}} \hat{\xi}$ is a $\hat{Q}$-martingale. The first assertion follows directly from Proposition 2.2 (a) noting that $\mathcal{M}^{\hat{H}} \hat{\xi}$ is positive by Lemma C.1. For the second assertion, we note that by Lemma A.1 (b) (ii), it is enough to show that $\mathcal{M}^{\hat{H}} \hat{\xi}$ is a $\left(Q^{\gamma}, \mathbb{F}^{\gamma}\right)$-martingale. To this end, by Proposition 2.2 (a) and (b), we may assume that $\Delta G(T)=0$ (using that $\hat{Q} \approx P$ ) and have to check that $\lim _{t \uparrow \uparrow T} \hat{\xi}(t)(1-\hat{H}(t))=0$. We distinguish two cases.

First, let $p \geq 1$ and fix $t \in[0, T)$. Then as $1-\hat{H}(t) \leq 1$,

$$
0 \leq \hat{\xi}(t)(1-\hat{H}(t)) \leq \hat{\xi}(t)(1-\hat{H}(t))^{1 / p}
$$

and it suffices to show that the right-hand side converges to 0 as $t \uparrow \uparrow T$. Using first the definitions of $\hat{\xi}$ and $\hat{H}$ in (C.1) and (3.14), then the definition of $a(\cdot, \cdot, 1)$ in (4.7), and finally the definition of $b(\cdot, \cdot, 1)$ in $(4.10)$,

$$
\begin{aligned}
\hat{\xi}(t)(1-\hat{H}(t))^{1 / p} & =\exp \left(\int_{0}^{t}\left(\phi^{\prime}(u) \frac{1}{p \sigma^{2}}\left(\mu-\phi^{\prime}(u) \hat{y}(u)\right)(1+\hat{y}(u))-\frac{\kappa^{G}(u)}{p}(1+\hat{y}(u))\right) \mathrm{d} u\right) \\
& =\exp \left(-\int_{0}^{t} \frac{\kappa^{G}(u)}{p}(1+\hat{y}(u))\left(1-\frac{\phi^{\prime}(u)}{\kappa^{G}(u)} \frac{1}{\sigma^{2}}\left(\mu-\phi^{\prime}(u) \hat{y}(u)\right)\right) \mathrm{d} u\right) \\
& =\exp \left(-\int_{0}^{t} \frac{\kappa^{G}(u)}{p}(1+\hat{y}(u)) a(u, \hat{y}(u), 1) \mathrm{d} u\right) \\
& =\exp \left(-\int_{0}^{t} \frac{\kappa^{G}(u)}{p} b(u, \hat{y}(u), 1) \mathrm{d} u\right) .
\end{aligned}
$$

By the representation of $n$ in (B.5), we have for $u \in[0, T)$,

$$
n(u, \hat{y}(u), p)=-\frac{1-p}{2 p^{2} \sigma^{2}} \mu^{2}+\frac{1-p}{2 p^{2} \sigma^{2}}\left(\phi^{\prime}(u) \hat{y}(u)-\mu\right)^{2}+\frac{1}{p} \kappa^{G}(u)(b(u, \hat{y}(u), 1)-1) .
$$

As the left-hand side as well as the first two summands on the right-hand side are integrable on $(0, T)$ by $\left(\right.$ B.16), we infer that $\int_{0}^{T} \kappa^{G}(u)|b(u, \hat{y}(u), 1)-1| \mathrm{d} u<\infty$. But $\Delta G(T)=0$ implies that

$$
\int_{0}^{T} \kappa^{G}(u) \mathrm{d} u=-\log (\Delta G(T))=\infty,
$$

and so the right-hand side of (C.11) converges to 0 as $t \uparrow \uparrow T$.

Second, let $p<1$ and fix $t \in[0, T)$. Using first the definitions of $\hat{\xi}$ and $\hat{H}$ in (C.1) and (3.14), and then the definition of $a$ in (4.7),

$$
\begin{aligned}
\hat{\xi}(t)(1-\hat{H}(t)) & =\exp \left(\int_{0}^{t}\left(\phi^{\prime}(u) \frac{1}{p \sigma^{2}}\left(\mu-\phi^{\prime}(u) \hat{y}(u)\right)(1+\hat{y}(u))-\kappa^{G}(u)(1+\hat{y}(u))\right) \mathrm{d} u\right) \\
& =\exp \left(-\int_{0}^{t} \kappa^{G}(u)(1+\hat{y}(u))\left(1-\frac{\phi^{\prime}(u)}{\kappa^{G}(u)} \frac{1}{p \sigma^{2}}\left(\mu-\phi^{\prime}(u) \hat{y}(u)\right)\right) \mathrm{d} u\right) \\
& =\exp \left(-\int_{0}^{t} \kappa^{G}(u)(1+\hat{y}(u)) a(u, \hat{y}(u), p) \mathrm{d} u\right) .
\end{aligned}
$$

Using the estimate

$$
(1+\hat{y}(u)) a(u, \hat{y}(u), p) \geq p\left(1+\frac{1}{p} \hat{y}(u)\right) a(u, \hat{y}(u), p)=p b(u, \hat{y}(u), p), \quad u \in[0, T)
$$

This article is protected by copyright. All rights reserved. 
we obtain

$$
0 \leq \hat{\xi}(t)(1-\hat{H}(t)) \leq \exp \left(-p \int_{0}^{t} \kappa^{G}(u) b(u, \hat{y}(u), p) \mathrm{d} u\right) .
$$

By the definition of $n$ in (4.12), we have for $u \in[0, T)$,

$$
n(u, \hat{y}(u), p)=-\frac{1-p}{2 p^{2} \sigma^{2}}\left(\phi^{\prime}(u) \hat{y}(u)\right)^{2}+\kappa^{G}(u)(b(u, \hat{y}(u), p)-1) .
$$

As the left-hand side as well as the first summand on the right-hand side are integrable on $(0, T)$ by (B.16), we infer that $\int_{0}^{T} \kappa^{G}(u)|b(u, \hat{y}(u), p)-1| \mathrm{d} u<\infty$. Combining this with (C.12) shows that the right-hand side of (C.13) converges to 0 as $t \uparrow \uparrow T$.

\section{References}

Adler, M., \& Detemple, J. 1988. On the optimal hedge of a nontraded cash position. J. Finance, 43, $143-153$.

Ausloos, M., Boveroux, P., Minguet, A., \& Vandewalle, N. 1998a. The crash of October 1987 seen as a phase transition: amplitude and universality. Physica A, 255, 201-210.

Ausloos, M., Boveroux, P., Minguet, A., \& Vandewalle, N. 1998b. How the financial crash of October 1997 could have been predicted. Eur. Phys. J. B, 4, 139-141.

Ausloos, M., Boveroux, P., Minguet, A., \& Vandewalle, N. 1999. Visualizing the log-periodic pattern before crashes. Eur. Phys. J. B, 9, 355-359.

Belak, C., Christensen, S., \& Menkens, O. 2014. Worst-case optimal investment with a random number of crashes. Statist. Probab. Lett., 90, 140-148.

Bouchaud, J.-P., Johansen, A., \& Sornette, D. 1996. Stock market crashes, precursors and replicas. J. Phys. I France, 6, 167-175.

Brée, D., \& Joseph, N. 2013. Testing for financial crashes using the log periodic power law model. Int. Rev. Financ. Anal., 30, 287-297.

Brunnermeier, M., \& Nagel, S. 2004. Hedge funds and the technology bubble. J. Finance, 59, 2013-2040.

Chacko, G., \& Viceira, L. 2005. Dynamic consumption and portfolio choice with stochastic volatility in incomplete markets. Rev. Financ. Stud., 18, 1369-1402.

Cox, A., \& Hobson, D. 2005. Local martingales, bubbles and option prices. Finance Stoch., 9, 477-492.

Fantazzini, D., \& Geraskin, P. 2013. Everything you always wanted to know about log-periodic power laws for bubble modeling but were afraid to ask. Eur. J. Finance, 19, 366-391.

Feigenbaum, J. 2001a. More on a statistical analysis of log-periodic precursors to financial crashes. Quant. Finance, 1, 527-532.

Feigenbaum, J. 2001b. A statistical analysis of log-periodic precursors to financial crashes. Quant. Finance, 1, 346-360.

Feigenbaum, J., \& Freund, P. 1996. Discrete scale invariance in stock markets before crashes. Int. J. Mod. Phys. B, 10, 3737-3745.

Gerhold, S., Guasoni, P., Muhle-Karbe, J., \& Schachermayer, W. 2014. Transaction costs, trading volume, and the liquidity premium. Finance Stoch., 18, 1-37.

Herdegen, M., \& Herrmann, S. 2014. A class of strict local martingales. Working paper SSRN:2402248.

Herdegen, M., \& Herrmann, S. 2016. Single jump processes and strict local martingales. Stoch. Proc. Appl., 126, 337-359.

This article is protected by copyright. All rights reserved. 
Heston, S., Loewenstein, M., \& Willard, G. 2007. Options and bubbles. Rev. Financ. Stud., 20, 359-390.

Jarrow, R., Protter, P., \& Shimbo, K. 2007. Asset price bubbles in complete markets. Pages 97-121 of: Fu, M., Jarrow, R., Yen, J.-Y., \& Elliott, R. (eds), Advances in Mathematical Finance. Applied and Numerical Harmonic Analysis. Boston: Birkhäuser.

Jarrow, R., Protter, P., \& Shimbo, K. 2010. Asset price bubbles in incomplete markets. Math. Finance, 20, 145-185.

Jiao, Y., \& Pham, H. 2011. Optimal investment with counterparty risk: a default-density model approach. Finance Stoch., 15, 725-753.

Johansen, A., \& Sornette, D. 1999a. Critical crashes. Risk, 12, 91-94.

Johansen, A., \& Sornette, D. 1999b. Modeling the stock market prior to large crashes. Eur. Phys. J. B, 9, 167-174.

Johansen, A., \& Sornette, D. 2001. Significance of log-periodic precursors to financial crashes. Quant. Finance, 1, 452-471.

Johansen, A., Ledoit, O., \& Sornette, D. 2000. Crashes as critical points. Int. J. Theor. Appl. Finance, 3, 219-255.

Kaizoji, T., \& Sornette, D. 2010. Bubbles and Crashes. In: Cont, R. (ed), Encyclopedia of Quantitative Finance. Hoboken: Wiley.

Kallsen, J. 2000. Optimal portfolios for exponential Lévy processes. Math. Meth. Oper. Res., 51, 357-374.

Kim, T., \& Omberg, E. 1996. Dynamic nonmyopic portfolio behavior. Rev. Financ. Stud., 9, 141-161.

Korn, R., \& Wilmott, P. 2002. Optimal portfolios under the threat of a crash. Int. J. Theor. Appl. Finance, 5, 171-187.

Kramkov, D., \& Schachermayer, W. 1999. The asymptotic elasticity of utility functions and optimal investment in incomplete markets. Ann. Appl. Probab., 9, 904-950.

Lim, T., \& Quenez, M.-C. 2011. Exponential utility maximization in an incomplete market with defaults. Electron. J. Probab., 16, 1434-1464.

Liu, J. 2007. Portfolio selection in stochastic environments. Rev. Financ. Stud., 20, 1-39.

Loewenstein, M., \& Willard, G. 2000. Rational equilibrium asset-pricing bubbles in continuous trading models. J. Econom. Theory, 91, 17-58.

Mossin, J. 1968. Optimal multiperiod portfolio policies. J. Bus., 41, 215-229.

Protter, P. 2005. Stochastic Integration and Differential Equations. 2nd edn. Stochastic Modelling and Applied Probability, vol. 21. Berlin: Springer.

Protter, P. 2013. A mathematical theory of financial bubbles. Pages 1-108 of: Paris-Princeton Lectures on Mathematical Finance 2013. Lecture Notes in Mathematics, vol. 2081. Berlin: Springer.

Scherbina, A., \& Schlusche, B. 2014. Asset price bubbles: a survey. Quant. Finance, 14, 589-604.

Seifried, F. 2010. Optimal investment for worst-case crash scenarios: a martingale approach. Math. Oper. Res., 35, 559-579.

Sornette, D., Woodard, R., Yan, W., \& Zhou, W.-X. 2013. Clarifications to questions and criticisms on the Johansen-Ledoit-Sornette financial bubble model. Physica A, 392, 4417-4428.

Temin, P., \& Voth, H. 2004. Riding the south sea bubble. Am. Econ. Rev., 94, 1654-1668.

v. Bothmer, H.-C. Graf, \& Meister, C. 2003. Predicting critical crashes? A new restriction for the free variables. Physica A, 320, 539-547.

Walter, W. 1998. Ordinary Differential Equations. Graduate Texts in Mathematics, vol. 182. Berlin: Springer.

This article is protected by copyright. All rights reserved. 